\title{
Algebraic Topology of Calabi-Yau Threefolds in Toric Varieties
}

\author{
Charles F. Doran and John W. Morgan
}

\begin{abstract}
We compute the integral homology (including torsion), the topological K-theory, and the Hodge structure on cohomology of Calabi-Yau threefold hypersurfaces and complete intersections in Gorenstein toric Fano varieties. The methods are purely topological.
\end{abstract}

\section{CONTENTS}

1. Preliminaries and statements of results 3

2. Calabi-Yau manifolds in toric varieties 7

2.1. Toric varieties: The affine case 8

2.2. Toric varieties: The general case 9

2.2.1. Refinements and induced maps: The affine case 10

2.2.2. Refinements and induced maps: The projective case 12

2.3. Lefschetz theorems and arithmetic genus of Calabi-Yau manifolds in toric varieties 14

2.3.1. Lefschetz theorems 15

2.3.2. Arithmetic genus 16

2.4. First computations of homology groups of Calabi-Yau threefolds $\quad 17$

2.4.1. Representation by divisors 20

3. Integral homologv of Calabi-Yau threefolds in toric varieties 20

3.1. The general set-up 21

3.2. The comparison of $\tilde{Y}_{1}$ and $Y_{7} \quad 22$

3.3. The comparison of $Y_{2}$ and $Y_{2} \quad 22$

3.4. The comparison of $Y$ and $Y$ - 25

3.5. The computation of $\rho_{*}: H_{1}(\hat{Y}) \rightarrow H_{1}(Y) \quad 28$

3.6. The computation of $\rho_{*}: H_{2}(\tilde{Y}) \rightarrow H_{2}(Y) \quad 29$

3.7. The computation of $\rho_{*}: H_{3}(Y) \rightarrow H_{3}(Y) \quad 30$

3.8. The tangent space to the space of polvnomial deformations 33

4. $K^{0}$ and $K^{1}$ of Calabi-Yau threefolds in terms of homology 34

References 36 
One of the most fruitful sources of Calabi-Yau threefolds is hypersurfaces, or more generally complete intersections, in toric varieties. This is especially true since there is a proposal for the mirror of any such Calabi-Yau three-fold. Usually the toric varieties associated to convex lattice polytopes are singular, causing the Calabi-Yau threefolds in them also to be singular, so that to get smooth Calabi-Yau threefolds we must resolve the ambient singularities and take the preimage in the resolution of the singular Calabi-Yau threefold. This can be done torically by the combinatorial device of taking a triangulation of the boundary of the convex lattice polytope defining the toric variety where the vertices of the triangulation are exactly the lattice points contained in the boundary of the polytope. In general, there will be many such triangulations of a given lattice polytope, leading to different ambient resolutions producing different families of Calabi-Yau threefolds associated with the original toric variety.

In spite of the existence of many different such resolutions of a given singular object, there are lattice-theoretic formulas for the Hodge numbers of these resolutions expressed in terms of the lattice polytope and its polar. Thus, the Hodge numbers of all the different resolutions coming from different triangulations are the same. The proofs of these combinatorial formulas rely on the Griffiths-Dwork method of computing Hodge numbers using residues of meromorphic differentials on the complement of the Calabi-Yau threefolds in the toric ambient space.

In this paper we study the resolution process from a more topological point of view. In the topological study of these objects, one treats both complete intersections and hypersurfaces simultaneously. This approach allows us to establish refined versions of the Hodge number counts described above. By directly considering the topology and algebraic geometry of the resolutions we compare the cohomology of the resolution with the cohomology of the singular hypersurface or complete intersection. From this we are able to see several things. The image of $H^{3}$ of the singular object in $H^{3}$ of the resolution is identified with the weight 3 quotient of $H^{3}$ of the singular object and hence is the same for all resolutions. The rest of $H^{3}$ of the resolution is of Hodge types $(2,1)$ and $(1,2)$. It is described as a sum of Hodge structures, summed over the edges of the polytope. The Hodge structure associated to an edge is the tensor product of the Hodge structures on $H^{1}$ of a smooth curve, contained in the closure of the two-dimensional toric orbit given by that edge, with a Hodge structure of type $(1,1)$ on the free abelian group with basis the set of lattice points interior to the edge. It follows that the rational Hodge structure on $H^{3}$ is independent of the choice of resolution. Also, under the identification of $H^{2,1}$ with the tangent space to the moduli space of complex structures, the image of $H^{2,1}$ of the singular object is identified with the tangent space of so called "polynomial deformations", i.e., the subspace of deformations obtained by varying the hypersurface or complete intersection in the toric variety. The non-polynomial deformations are then accounted for by the curves associated to the edges of the polytope and the lattice points in the interiors of these edges. In this way, we recover the so called "correction term" describing the dimension of the non-polynomial deformations directly from the resolution description.

It also follows from the resolution description that every class in $H^{2}$ of the resolution is Poincaré dual to a divisor, a toric divisor. As a consequence, the Hodge type of $H^{2}$ of the resolution is $(1,1)$. We are able to recover the combinatorial 
formula for the rank of this group from an understanding of the resolutions of singularities.

Besides allowing us to enhance the combinatorial counts of Hodge numbers to results about Hodge structures and allowing us to see directly in terms of subvarieties and other topological objects the sources of these homology groups, there are other advantages to our topological approach. It permits us to establish results over the integers, and these of course lead to results in topological K-theory. Since $\mathrm{K}$-theory is the repository for the most refined conjectures about mirror symmetry, understanding of the integral homology and cohomology is crucial for more refined tests of mirror symmetry. ${ }^{1}$ While this paper establishes all the necessary topology results over $\mathbb{Z}$ in order to examine the mirror symmetry proposals (see, for example, [DM]), we do not explore these issues here; they will be taken up in another paper.

\section{Preliminaries and statements of results}

Let $N$ be a lattice of dimension $n, \Delta \subset N_{\mathbb{R}}=N \otimes_{\mathbb{Z}} \mathbb{R}$ be a reflexive polytope, and $\mathbb{P}_{\Delta}$ the associated toric variety. Denote by $V=V(\Delta)$ the set of vertices of $\Delta$. If $n=4$, denote by $Y$ the vanishing locus of a generic section of $\mathcal{O}\left(D_{\infty}\right)$ where

$$
D_{\infty}=\sum_{v \in V} \overline{\mathcal{O}_{v}}
$$

and $\mathcal{O}_{v}$ denotes the codimension-one torus orbit in $\mathbb{P}_{\Delta}$ associated to the vertex $v$.

Here are the standard facts about toric varieties determined by reflexive polytopes [CK $\S 3.5]$

Lemma 1.1. Suppose that $\Delta \subset N_{\mathbb{R}}$ is a reflexive polytope. Let $D_{\infty} \subset \mathbb{P}_{\Delta}$ be the Weil divisor

$$
D_{\infty}=\sum_{v \in V(\Delta)} \overline{\mathcal{O}}_{v}
$$

The complete variety $\mathbb{P}_{\Delta}$ is Cohen-Macaulay. The divisor $D_{\infty}$ is a Cartier divisor in $\mathbb{P}_{\Delta}$. The line bundle $\mathcal{O}\left(D_{\infty}\right)$ is very ample, and anti-canonical.

If $n>4$ suppose further that we have a NEF partition $V=V_{1} \amalg V_{2} \amalg \cdots \amalg V_{n-3}$. In this case, suppose

$$
D_{i}=\sum_{v \in V_{i}} \overline{\mathcal{O}_{v}}
$$

are ample divisors in $\mathbb{P}_{\Delta}$, and let $Y \subset \mathbb{P}_{\Delta}$ be the complete intersection of generic sections of $\mathcal{O}\left(D_{i}\right), 1 \leq i \leq n-3$.

For both the case when $Y$ is a hypersurface and a complete intersection, we denote by $Y_{i} \subset Y$ the subvariety which is the intersection of $Y$ with the union of the torus orbits of codimension $\leq i$.

For $\mathcal{T}$ a maximal triangulation of $\partial \Delta$, i.e., one whose set of vertices is $N \cap \partial \Delta$, let $\mathbb{P}_{\mathcal{T}}$ be the toric variety associated to the fan given by the cones over the simplices of $\mathcal{T}$ and let

$$
\rho: \mathbb{P}_{\mathcal{T}} \rightarrow \mathbb{P}_{\Delta}
$$

be the morphism of toric varieties associated to the inclusion of this fan into the fan determined by $\Delta$, and define $\widetilde{Y}=\rho^{-1}(Y)$.

\footnotetext{
${ }^{1}$ In BK, Batyrev and Kreuzer use a different method to study the integral cohomology of Calabi-Yau threefold hypersurfaces in toric varieties and obtain related results, including some computations suggesting a role played by torsion in cohomology in mirror symmetry.
} 
The variety $\tilde{Y} \subset \mathbb{P}_{\mathcal{T}}$ is a (smooth) Calabi-Yau three-fold, and this construction of Calabi-Yau threefolds as complete intersections in Gorenstein toric Fano varieties, due to Batyrev and Borisov, is by far the richest known source of compact CalabiYau threefolds. In the hypersurface case alone, where the requisite combinatorial data consists simply of a four dimensional reflexive polytope $\Delta$, the classification of such polytopes, due to Kreuzer and Skarke KS, results in more than 400 million example Calabi-Yau threefold hypersurface families.

Our goal in this paper is a complete understanding of the integral (co)homology, Hodge structure, and topological K-theory of such Calabi-Yau threefolds $\widetilde{Y}$. The approach taken is thoroughly algebro-topological by looking directly at this resolution.

The Hodge diamonds of the Calabi-Yau threefolds $\widetilde{Y}$ look like this

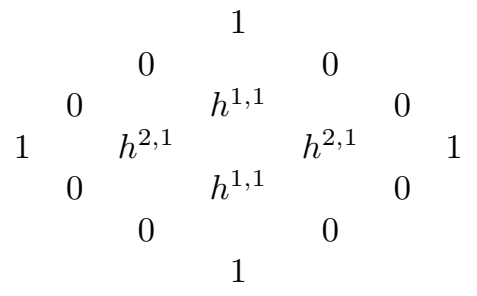

and there are combinatorial formulas expressing the Hodge numbers $h^{1,1}$ and $h^{2,1}$. According to Bat, BB], these formulas involve the distribution of the points of $\Delta \cap N$ in the various faces of $\Delta$ and its polar polytope $\Delta^{\circ}$ and also, in the case $n>4$, the NEF partition/dual NEF partition. Let $E, F$, and $G$ denote respectively the sets of edges, two-faces, and three-faces of $\partial \Delta$, and $E^{\circ}, F^{\circ}$, and $G^{\circ}$ the same for $\partial \Delta^{\circ}$. For simplicity, let us consider formulas for $h^{1,1}$ and $h^{2,1}$ in the case of Calabi-Yau threefold hypersurfaces:

$$
\begin{gathered}
h^{1,1}=\ell(\Delta)-5-\sum_{g \in G} \ell^{*}(g)+\sum_{f \in F} \ell^{*}(f) \ell^{*}\left(f^{\vee}\right) \\
h^{2,1}=\ell\left(\Delta^{\circ}\right)-5-\sum_{v \in V} \ell^{*}\left(v^{\vee}\right)+\sum_{e \in E} \ell^{*}(e) \ell^{*}\left(e^{\vee}\right) .
\end{gathered}
$$

Here, $\ell(\Delta)$ is the cardinality of $N \cap \Delta$, and $\ell\left(\Delta^{\circ}\right)$ is defined analogously with respect to the dual lattice $\operatorname{Hom}(N, \mathbb{Z})$. For a face $\alpha$ of $\partial \Delta, \ell^{*}(\alpha)$ denotes the number of lattice points in the relative interior of $\alpha$, and $\alpha^{\vee}$ is the dual face to $\alpha$ in $\partial \Delta^{\circ}$.

Although these formulas were used by Batyrev to check predictions from mirror symmetry on the level of Hodge diamonds, there are two difficulties with this approach which prevent the broader applicability of its methods. First, for many applications to physical questions one would really like to have a direct geometric construction of the topological cycles generating the cohomology, especially over $\mathbb{Z}$. One would like a complete description of the Hodge structure on cohomology, rather than just the Hodge numbers, and to have it expressed directly in terms of these geometric representatives. Also, there is the issue of relating these Hodge structures for $\tilde{Y}$ to those of the original singular variety $Y$, and seeing how, if at all, the answer depends on the choice of maximal triangulation $\mathcal{T}$.

We begin by describing our results in the case of $H^{2}(\widetilde{Y})$, where the geometric representatives are more similar to those in the literature, though the algebrotopological methods of proof are quite different. 
As is well known, all of $H^{2}(\widetilde{Y})$ is spanned by algebraic cycles and hence the Hodge decomposition of $H^{2}(\widetilde{Y})$ is all of type $(1,1)$. We establish in fact

THEOREM 1.2. Let $\Delta$ be a reflexive polytope and $\mathcal{T}$ a maximal triangulation of $\partial \Delta$. If $\operatorname{dim}(\Delta)=4$, let $Y \subset \mathbb{P}_{\Delta}$ be a generic section of $\mathcal{O}\left(D_{\infty}\right)$. If $\operatorname{dim} \Delta>4$ suppose that we have a NEF partition of $V(\Delta)$ with associated divisors $D_{i}$, with the divisors $D_{i} \subset \mathbb{P}_{\Delta}$ being ample. Let $\rho: \mathbb{P}_{\mathcal{T}} \rightarrow \mathbb{P}_{\Delta}$ be the natural resolution and let $\tilde{Y}=\rho^{-1}(Y)$. We have the following results.

(1) In the hypersurface case,

$$
\operatorname{rank} H^{2}(\tilde{Y})=\sum_{e \in E} \ell^{*}(e)+\sum_{f \in F} \ell^{*}(f) \cdot\left(\ell^{*}\left(f^{\vee}\right)+1\right)+\# V-4 .
$$

(2) The Hodge structure on $H^{2}(\widetilde{Y})$ is of type $(1,1)$. Every integral class in $H^{2}(\widetilde{Y} ; \mathbb{Z})$ is an integral linear combination of classes Poincaré dual to irreducible components of toric divisors in $\tilde{Y}$, i.e., divisors in $\tilde{Y}$ that are irreducible components of the intersection of $\widetilde{Y}$ with the closure of a codimension-one torus orbit in $\mathbb{P}_{\mathcal{T}}$.

(3) The mixed Hodge structure on $H^{2}(Y)$ is a pure Hodge structure of weight 2 and Hodge type $(1,1)$.

REMARK 1.3. Let us describe the irreducible components of the intersection of $\widetilde{Y}$ with the closures of a codimension-one torus-orbit. These orbits are indexed by the vertices of $\mathcal{T}$, i.e., $N \cap \partial \Delta$. For each $\ell \in \partial \Delta \cap N$, there is a divisor $R(\ell) \subset \widetilde{Y}$. Let $\widetilde{\mathcal{O}}_{\ell}$ be the associated torus orbit in $\mathbb{P}_{\mathcal{T}}$. Then $R(\ell)$ is the closure of the intersection $\widetilde{Y} \cap \widetilde{\mathcal{O}}_{\ell}$.

When $\ell \in V$ is a vertex, $R(\ell)$ is an irreducible complex surface projecting birationally onto the surface $Y \cap \overline{\mathcal{O}_{\ell}}$ in $Y$.

When $\ell \in \stackrel{\circ}{e}$ lies in the interior of an edge $e \in E$, letting $\widehat{Z}(e)$ denote the smooth and complete curve $Y \cap \overline{\mathcal{O}_{e}}, R(\ell)$ is a ruled surface over the curve $\widehat{Z}(e)$ with generic fiber a smooth rational curve $\mathbb{P}^{1}(\ell)$. In particular, $H_{3}(R(\ell))=H_{1}(\widehat{Z}(e)) \otimes$ $H_{2}\left(\mathbb{P}^{1}(\ell)\right)$. The union over all $\ell \in e^{\circ}$ of the $R(\ell)$ produces a fibration where the

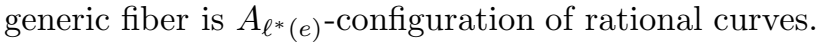

Finally, when $\ell \in f$ for a 2 -face $f \in F, R(\ell)$ is a disjoint union of $\#\left(Y \cap \mathcal{O}_{f}\right)$ copies of a surface and maps to $Y \cap \mathcal{O}_{f}$ in $Y$.

The results in the hypersurface case described in part (1) of the theorem imply an expression for $h^{1,1}(\widetilde{Y})$. This agrees with that from formula (1.1), since

$$
\ell(\Delta)=1+\# V+\sum_{e \in E} \ell^{*}(e)+\sum_{f \in F} \ell^{*}(f)+\sum_{g \in G} \ell^{*}(g) .
$$

There is an analogue of part (1) in the complete intersection case, and corresponding expressions for $h^{1,1}(\widetilde{Y})$ in the complete intersection case (see Section 2.4 below and BB $\S 8]$.

We now describe our corresponding results for $H^{3}(\tilde{Y})$ :

THEOREM 1.4. In the hypersurface case or in the complete intersection case when all the divisors $D_{a} \subset \mathbb{P}_{\Delta}$ are ample we have the following results. 
(1) The image of

$$
\rho_{*}: H_{3}(\widetilde{Y}) \rightarrow H_{3}(Y)
$$

is equal to the image of

$$
H_{3}\left(Y_{2}\right) \rightarrow H_{3}(Y)
$$

(2) Let $\mathcal{A}_{\ell^{*}(e)}$ be the lattice (with symmetric bilinear pairing) associated to the root system $A_{\ell^{*}(e)}$. Then

$$
\operatorname{Ker} \rho_{*}: H_{3}(\widetilde{Y}) \rightarrow H_{3}(Y)
$$

is identified with

$$
\bigoplus_{e \in E} H_{1}(\widehat{Z}(e)) \otimes \mathcal{A}_{\ell^{*}(e)} .
$$

Under this isomorphism, the restriction to Ker $\rho_{*}$ of the usual homological intersection pairing is identified with the direct sum of the tensor products of the intersection pairings on $H_{1}(\widehat{Z}(e))$ with the natural pairing on $\mathcal{A}_{\ell^{*}(e)}$.

It follows that

$$
\text { Image }\left(H_{3}(\tilde{Y} ; \mathbb{Z}) \rightarrow H_{3}(Y ; \mathbb{Z})\right)
$$

is independent of the choice of maximal triangulation $\mathcal{T}$. By general results in mixed Hodge theory Del] in fact

$$
\text { Image }\left(H_{3}(\tilde{Y} ; \mathbb{Q}) \rightarrow H_{3}(Y ; \mathbb{Q})\right)
$$

is $W_{-3} \subset H_{3}(Y ; \mathbb{Q})$ under the mixed Hodge structure on $H_{3}(Y ; \mathbb{Q})$.

COROLlary 1.5. We have the following exact sequence of $\mathbb{Q}$-Hodge structures of weight three:

$$
0 \rightarrow \frac{H^{3}(Y ; \mathbb{Q})}{W_{2} H^{3}(Y ; \mathbb{Q})} \rightarrow H^{3}(\tilde{Y} ; \mathbb{Q}) \rightarrow \bigoplus_{e \in E} H^{1}(\widehat{Z}(e)) \otimes \mathcal{A}_{\ell^{*}(e)}^{*} \rightarrow 0,
$$

where the first term is a pure weight three quotient of $H^{3}(Y ; \mathbb{Q})$. The sequence splits so that the Hodge structure on $H^{3}(\widetilde{Y} ; \mathbb{Q})$ is isomorphic to the direct sum of this pure weight three Hodge structure and the direct sum of tensor products of Hodge structures from the third term. The factor $\mathcal{A}_{\ell^{*}(e)}^{*}$ is defined to be of type $(1,1)$, so that the third term has Hodge types $(2,1)$ and $(1,2)$.

COROLLARY 1.6. The isomorphism type of the $\mathbb{Q}$-Hodge structure on $H^{3}(\widetilde{Y})$ is independent of the choice of resolution $\widetilde{Y}$ of $Y$ (corresponding to the choice of maximal triangulation $\mathcal{T}$ of $\partial \Delta$ ).

The last terms in formulas (1.1) and (1.2) are typically called the "correction terms", and without these terms the formulas describe the so-called toric part $h_{\text {toric }}^{1,1}$ of $h^{1,1}(\widetilde{Y})$ and the polynomial part $h_{\text {poly }}^{2,1}$ of $h^{2,1}(\widetilde{Y})$. The number $h_{\text {toric }}^{1,1}$ is the dimension of the subspace of $H^{1,1}(\widetilde{Y} ; \mathbb{C})$ generated by the restriction to $\widetilde{Y}$ of the $T_{N}$-invariant divisors on $\mathbb{P}_{\mathcal{T}}$. Thus, by parts (2) and (3) of Theorem 1.2 the difference between $h_{\text {toric }}^{1,1}$ and $h^{1,1}$ is given by $\ell^{*}(f) \cdot \ell^{*}\left(f^{\vee}\right)$ and reflects the fact that, for $\ell \in \stackrel{\circ}{f}, R(\ell)$ has $\ell^{*}\left(f^{\vee}\right)+1$ components. The number $h_{\text {poly }}^{2,1}$ counts the dimension of the space of polynomial deformations of $\tilde{Y} \subset \mathbb{P}_{\mathcal{T}}$, i.e., the space of deformations of complex structure of $\widetilde{Y}$ determined by the hypersurfaces in the 
anti-canonical linear system $\left|-K_{\mathbb{P}_{\mathcal{T}}}\right|$, or equivalently deformations of $\tilde{Y}$ induced by taking the preimages under $\rho$ of deformations of $Y \subset \mathbb{P}_{\Delta}$.

Corollary 1.7. The sequence in Corollary 1.5 is compatible with the subspace of polynomial deformations of $\tilde{Y}$ in the sense that the subspace $\left(H^{3}(Y) / W_{2} H^{3}(Y)\right)^{2,1}$ of $H^{2,1}(\widetilde{Y})$ is the tangent space to the space of polynomial deformations of $\widetilde{Y}$.

The methods used in establishing the above results on (co)homology of the Calabi-Yau threefolds $\widetilde{Y}$ involve both "standard" local decomposition techniques, guided by the toric structure on $\mathbb{P}_{\mathcal{T}}$, and some combinatorial topology about the two-faces of the reflexive polytope $\Delta$ itself.

The methods apply not only to integral cohomology but also to $K$-theory. To see this we use the direct link between topological K-theory and integral homology (including torsion). A careful analysis of the 7-dimensional stage $B S U^{(7)}$ of the Postnikov tower for $B S U$ leads us to the following technical result.

Theorem 1.8. Let $M$ be a closed, oriented 6-manifold. The reduced even $K$ group $\widetilde{K^{0}}(M)$ is isomorphic to

$$
\left\{\left(c_{1}, c_{2}, c_{3}\right) \in H^{2}(M ; \mathbb{Z}) \oplus H^{4}(M ; \mathbb{Z}) \oplus H^{6}(M ; \mathbb{Z}) \mid S q^{2} c_{2}=\left[c_{3}\right]_{2}+c_{1} c_{2}+c_{1}^{3}\right\}
$$

where the isomorphism is given by taking the 1st, 2nd, and 3rd Chern class. The odd $K$-group $K^{1}(M)=\widetilde{K^{0}}(\Sigma M)$ is

$$
H^{1}(M ; \mathbb{Z}) \times\left[\Sigma M, B S U^{(7)}\right]
$$

and we have an exact sequence

$$
0 \rightarrow H^{5}(M ; \mathbb{Z}) \rightarrow\left[\Sigma M, B S U^{(7)}\right] \rightarrow H^{3}(M ; \mathbb{Z}) \rightarrow 0,
$$

where the extension is the pullback of the extension

$$
0 \rightarrow H^{5}(M ; \mathbb{Z}) \rightarrow A \rightarrow H^{3}(M ; \mathbb{Z}) \rightarrow 0
$$

with extension class given by the $S q^{2}$ map

$$
H^{3}(M ; \mathbb{Z} / 2 \mathbb{Z}) \rightarrow \frac{H^{5}(M ; \mathbb{Z})}{2 H^{5}(M ; \mathbb{Z})}=H^{5}(M ; \mathbb{Z} / 2 \mathbb{Z})
$$

For Calabi-Yau threefolds, independent of whether or not the manifolds are constructed as hypersurfaces or complete intersections in a toric variety, we establish the following.

Corollary 1.9. Let $M$ be a Calabi-Yau threefold. The topological K-groups of $M$ are expressed in terms of the integral homology groups of $M$ :

$$
K^{0}(M) \cong \mathbb{Z} \oplus H^{2}(M ; \mathbb{Z}) \oplus H^{4}(M ; \mathbb{Z}) \oplus 2 \cdot H^{6}(M ; \mathbb{Z}),
$$

and

$$
K^{1}(M) \cong H^{1}(M ; \mathbb{Z}) \oplus H^{3}(M ; \mathbb{Z}) \oplus H^{5}(M ; \mathbb{Z})
$$

\section{Calabi-Yau manifolds in toric varieties}

This section lays out notations and basic results from toric geometry which we will use in subsequent sections. Good references for many of these are the books CK, Ful].

Let $N \simeq \mathbb{Z}^{n}$ be a lattice, $M=\operatorname{Hom}(N, \mathbb{Z})$ the dual lattice, $N_{\mathbb{R}}=N \otimes_{\mathbb{Z}} \mathbb{R}$ the associated real vector space, and $T_{N}=N \otimes_{\mathbb{Z}} \mathbb{C}^{*}$ the associated complex torus. 
2.1. Toric varieties: The affine case. Let $c$ be a rational polyhedral cone in $N$. We say that $c$ is strongly convex if $c \cap(-c)=\{0\}$. The dual cone $\check{c}$ of a strongly convex rational polyhedral cone $c$ is

$$
\check{c}=\left\{x \in M_{\mathbb{R}} \mid\langle x, c\rangle \geq 0\right\} .
$$

Define the associated open affine toric variety by

$$
U(c, N)=\operatorname{Spec} \mathbb{C}[M \cap \check{c}] .
$$

The action $T_{N} \times U(c, N) \rightarrow U(c, N)$ is dual to the morphism

$$
\begin{aligned}
\mathbb{C}[M \cap \check{c}] & \rightarrow \mathbb{C}[M \cap \check{c}] \otimes_{\mathbb{C}} \mathbb{C}[M] \\
\chi^{m} & \mapsto\left(\chi^{m} \otimes \chi^{m}\right)
\end{aligned}
$$

where the characters $\chi^{m}: T_{N} \rightarrow \mathbb{C}^{*}$ for $m \in M \cap \check{c}$ generate the $\mathbb{C}$-algebra $\mathbb{C}[M \cap \check{c}]$. Since $c$ is strongly convex, $M \cap \check{c}$ spans $M_{\mathbb{R}}$ as a real vector space and hence this action has a free $T_{N}$-orbit that is open and dense.

The faces of $c$ are themselves polyhedral cones and the set of faces is a poset under inclusion, which we denote by $f \prec f^{\prime}$. Consider the 1-dimensional faces $r \prec c$ of the cone $c$. The generators of $c$, denoted as a set by $\mathcal{G}(c)$, are the generators of the semigroups $r \cap N$ as $r$ runs through all the 1-dimensional faces of $c$. We denote by $\stackrel{\circ}{c}$ the relative interior

$$
\stackrel{\circ}{c}=c \backslash \bigcup_{\{f \prec c \mid f \neq c\}} f .
$$

Let $f$ be a face of $c$. We define $K_{f} \subset M \cap \check{c}$ as follows:

$$
K_{f}=\{m \in M \cap \check{c} \mid\langle m, f\rangle=0\} .
$$

Corresponding to the face $f$ of $c$ there is a closed $T_{N}$-invariant subset in $U(c, N)$ defined by

$$
\mathcal{Z}_{f}=\bigcap_{m \in(M \cap \check{c}) \backslash K_{f}}\left\{\chi^{m}=0\right\} .
$$

This subset is the closure in $U(c, N)$ of the $T_{N}$-orbit $\mathcal{O}_{f}$ (sometimes denoted $\mathcal{O}_{f, N}$ if we need to specify the lattice $N$ to avoid confusion). This orbit is the open subset of $\mathcal{Z}_{f}$ consisting of all points $z \in \mathcal{Z}_{f}$ with the property that for every $m \in K_{f} \chi^{m}(z) \neq 0$. The union of the orbits $\mathcal{O}_{f}$, as $f$ ranges over all the faces of $c$, is $U(c, N)$. The closure $\overline{\mathcal{O}}_{f}$ is the union of $\mathcal{O}_{f^{\prime}}$ as $f^{\prime}$ ranges over all faces of $c$ satisfying $f \prec f^{\prime}$. The open dense orbit corresponds to the face of $c$ given by the point $\{0\}$. At the other extreme, there is exactly one closed orbit; it is $\mathcal{O}_{c}$. We denote by $U^{*}(c, N)$ the complement of this orbit. For maximal dimensional cones we have the following result about the closed orbit and also about the structure of the toric variety $U(c, N)$.

Lemma 2.1. Suppose that $c$ spans $N_{\mathbb{R}}$, i.e. the dimension of $c$ is $n$. The orbit $\mathcal{O}_{c}$ associated to the cone $c$ itself is a fixed point of the $T_{N}$-action. If $c$ is simplicial, then $U(c, N) \simeq \mathbb{C}^{n} / \Gamma$, where

$$
\Gamma=N / \mathbb{Z}-\operatorname{span}(\mathcal{G}(c)) .
$$

Now let us consider the case when $c$ is not of maximal dimension. Let $\mathbb{R}$-span $(c) \subset$ $N_{\mathbb{R}}$ be the linear span of the cone $c$ as a vector subspace. The dimension of $c$ is defined to be the dimension of $\mathbb{R}-\operatorname{span}(c)$. We define $N_{c}=N \cap \mathbb{R}$-span $(c)$. Of course, 
$c$ is a polyhedral cone in $\left(N_{c}\right)_{\mathbb{R}}$. We denote by $V(c)$ the toric variety $U\left(c, N_{c}\right)$. According to Lemma 2.1 there is a unique fixed point, denoted $0_{c}$ for the $T_{N_{c}}$-action on $V(c)$.

Dual to the inclusion $N_{c} \hookrightarrow N$ is the surjection $M \rightarrow M_{c}$, where $M_{c}=$ $\operatorname{Hom}\left(N_{c}, \mathbb{Z}\right)$. Denote by $K_{c}$ the kernel of $M \rightarrow M_{c}$. We have in fact the (noncanonically split) exact sequence of abelian semigroups

$$
0 \rightarrow K_{c} \rightarrow M \cap \check{c} \rightarrow M_{c} \cap \check{c} \rightarrow 0 .
$$

This gives rise to ring homomorphisms

$$
\mathbb{C}\left[K_{c}\right] \rightarrow \mathbb{C}[M \cap \check{c}] \rightarrow \mathbb{C}\left[M_{c} \cap \check{c}\right] .
$$

Since $K_{c}$ is identified with $\operatorname{Hom}\left(N / N_{c}, \mathbb{Z}\right)$, and since $N_{c} \subset N$ is a direct summand, we have $T_{N / N_{c}}=T_{N} / T_{N_{c}}$. Thus, we the dual morphisms are

$$
V(c) \rightarrow U(c, N) \rightarrow T_{N} / T_{N_{c}} .
$$

The map $U(c, N) \rightarrow T_{N} / T_{N_{c}}$ is a fibration and $V(c)$ is the fiber over the identity element. Since $N_{c}$ is a direct summand of $N$, there is a (non-canonical) splitting $T_{N} / T_{N_{c}} \hookrightarrow T_{N}$. Restricting the action $T_{N} \times U(c, N) \rightarrow U(c, N)$ to the image of the splitting determines an isomorphism

$$
V(c) \times T_{N} / T_{N_{c}} \stackrel{\psi}{\rightarrow} U(c, N) .
$$

We have the product action of $T_{N_{c}} \times\left(T_{N} / T_{N_{c}}\right)$ on $V(c) \times\left(T_{N} / T_{N_{c}}\right)$. The splitting identifies this product torus with $T_{N}$ and with this identification the isomorphism $\psi$ is equivariant. The closed orbit $\mathcal{O}_{c}$ in $U(c, N)$ is the product of $0_{c} \in V(c)$ with $T_{N} / T_{N_{c}}$.

Lemma 2.2. There is a $\mathbb{C}^{*}$-action on $V(c)$ whose the only fixed point is $0_{c}$ and such that for any $x \in V(c), \lim _{\lambda \rightarrow 0} \lambda \cdot x=0_{c}$. Restricting to $\mathbb{R}^{+} \subset \mathbb{C}^{*}$ produces the natural contraction action of a real cone-structure on $V(c)$ with cone point $0_{c}$.

Proof. Let $n \in N_{c}$ be a primitive vector contained in the interior of the cone c. Associated to this vector is an embedding $\imath_{n}: \mathbb{C}^{*} \hookrightarrow N_{c} \otimes_{\mathbb{Z}} \mathbb{C}^{*}$. We claim that for any $x \in V(c)$,

$$
\lim _{\lambda \rightarrow 0} \imath_{n}(\lambda) \cdot x
$$

is the fixed point $0_{c}$. The reason is that if $u \in M_{c} \backslash\{0\}$ is $\geq 0$ on the cone $c$, then $\langle u, n\rangle>0$ and hence

$$
\lim _{\lambda \rightarrow 0} \chi^{u}\left(\imath_{n}(\lambda) \cdot x\right)=0 .
$$

This means that the orbits of the $\mathbb{R}^{+} \subset \mathbb{C}^{*}$ action give a flow on $V(c)$ contracting it to the cone point.

2.2. Toric varieties: The general case. A $\operatorname{fan} \mathcal{F}$ in $N_{\mathbb{R}}$ is a finite collection of strongly convex rational polyhedral cones in $N_{\mathbb{R}}$ such that

(1) If $c \in \mathcal{F}$, then every face of $c$ is also in $\mathcal{F}$.

(2) If $c_{1}, c_{2} \in \mathcal{F}$, then $c_{1} \cap c_{2}$ is a face of each of $c_{1}$ and $c_{2}$.

The support of a fan $\mathcal{F}$ is the set

$$
|\mathcal{F}|=\bigcup_{c \in \mathcal{F}} c \subset N_{\mathbb{R}} .
$$

We obtain the toric variety $\mathbb{P}_{\mathcal{F}}$ from a fan $\mathcal{F} \subset N_{\mathbb{R}}$ by gluing together the affine $T_{N}$-spaces $U(c, N)$ for all $c \in \mathcal{F}$, where $U\left(c_{1}, N\right)$ and $U\left(c_{2}, N\right)$ are glued 
together along $U\left(c_{1} \cap c_{2}, N\right)$. (To avoid ambiguity arising from choice of lattice, we sometimes denote the toric variety associated with a fan $\mathcal{F} \subset N_{\mathbb{R}}$ by $\mathbb{P}_{\mathcal{F}, N}$.) The variety $\mathbb{P}_{\mathcal{F}}$ is complete if and only if the fan $\mathcal{F}$ is complete, i.e., $|\mathcal{F}|=N_{\mathbb{R}}$. By the proposition on integral closure in Ful p. 29], irreducible toric varieties are normal, and hence nonsingular through codimension two. It follows from the affine case that there is a one-to-one correspondence between the $T_{N}$-orbits and cones of $\mathcal{F}$, with the adherence relation being the opposite of the face relation; i.e., for cones $c_{1}$ and $c_{2}$,

$$
\mathcal{O}_{c_{1}} \subset \overline{\mathcal{O}}_{c_{2}} \Leftrightarrow c_{2} \prec c_{1} .
$$

Given a strongly convex rational polyhedral cone $c$, we define $\mathcal{F}_{c}$ to be the fan consisting of $c$ and all its faces. In this case we have $\mathbb{P}_{\mathcal{F}_{c}}=U(c, N)$. Also considering $c$ as a polyhedral cone in $\left(N_{c}\right)_{\mathbb{R}}$, let $\mathcal{F}_{c}\left(N_{c}\right)$ denote the fan in $\left(N_{c}\right)_{\mathbb{R}}$ consisting of $c$ and all its faces. We have $\mathbb{P}_{\mathcal{F}_{c}\left(N_{c}\right)}=U\left(c, N_{c}\right)$. For a general fan $\mathcal{F} \subset N_{\mathbb{R}}$ we have $\mathbb{P}_{\mathcal{F}}=\bigcup_{c \in \mathcal{F}} U(c, N)$.

One natural way that complete fans arise is from convex lattice polytopes $\Delta \subset N_{\mathbb{R}}$. For us a convex lattice polytope contains the origin in its interior and has points of $N$ as its vertices. We denote by $V(\Delta)$ its set of vertices, by $E=E(\Delta)$ its set of edges and by $F=F(\Delta)$ its set of two-faces. We define the fan $\mathcal{F}(\Delta)$ associated $^{2}$ with $\Delta$ consisting of cones over the faces of $\partial \Delta$ (by convention we include as a face the empty face whose cone is the origin), i.e., over a face $\gamma$ consider the cone

$$
c(\gamma)=\left\{\lambda\left(x-x^{\prime}\right) \mid x \in \Delta, x^{\prime} \in \gamma, \lambda \geq 0\right\} \subset N_{\mathbb{R}} .
$$

We define the associated toric variety

$$
\mathbb{P}_{\Delta}=\mathbb{P}_{\mathcal{F}(\Delta)}
$$

For any face $\gamma$ of $\partial \Delta$ we denote by $\mathcal{O}_{\gamma}$ the $T_{N \text {-orbit }} \mathcal{O}_{c(\gamma)}$, by $U(\gamma, N)$ the affine toric variety $U(c(\gamma), N)$, by $N_{\gamma}$ the sublattice $N_{c(\gamma)}$, and by $V(\gamma)$ the affine toric variety $V(c(\gamma))$.

A notation we shall use throughout is the following: If $\gamma$ is a face of a lattice polytope then $\ell^{*}(\gamma)$ denotes the number of lattice points contained in the relative interior of the face; i.e,

$$
\ell^{*}(\gamma)=\#\{N \cap \stackrel{\circ}{\gamma}\}
$$

2.2.1. Refinements and induced maps: The affine case. We say that a fan $\mathcal{F}^{\prime}$ refines the fan $\mathcal{F}$ if each cone of $\mathcal{F}^{\prime}$ is contained in a cone of $\mathcal{F}$ and that the supports of $\mathcal{F}^{\prime}$ and $\mathcal{F}$ are the same.

Let's begin with the case of refining a single cone. Fix a strongly convex rational polyhedral cone $c$ and suppose that $\mathcal{F}_{c}^{\prime}$ is a fan refining $\mathcal{F}_{c}$.

LEMma 2.3. There is a $T_{N}$-invariant morphism $\rho: \mathbb{P}_{\mathcal{F}_{c}^{\prime}} \rightarrow U(c, N)$ which is a birational isomorphism.

Proof. Let $c^{\prime}$ be a cone of $\mathcal{F}_{c}^{\prime}$. Since $c^{\prime} \subset c$, it follows that $\check{c} \subset \check{c}^{\prime}$. The map $U\left(c^{\prime}, N\right) \rightarrow U(c, N)$ is dual to the inclusion

$$
\mathbb{C}[M \cap \check{c}] \rightarrow \mathbb{C}\left[M \cap \check{c}^{\prime}\right]
$$

\footnotetext{
${ }^{2}$ Our associated fan is not what is referred to in $\mathbf{C K}$ as the normal fan of $\Delta$; in our notation, the normal fan of $\Delta \subset N_{\mathbb{R}}$ is a fan in $M_{\mathbb{R}}$.
} 
It is easy to see that these affine maps are $T_{N}$-equivariant and are fit together to define the map $\rho$ as given in the statement.

We wish to describe the pre-image of the orbit $\mathcal{O}_{c}$ under the map $\rho: \mathbb{P}_{\mathcal{F}_{c}^{\prime}} \rightarrow$ $U(c, N)$. Because of the $T_{N}$-equivariance, this pre-image is a union of $T_{N}$-orbits. In order to describe this map, let us introduce the dual cell decomposition $\Sigma\left(\mathcal{F}_{c}^{\prime}\right)$ of the refinement. There is one cell $\sigma^{\prime}$ dual to each cone $c^{\prime}$ of $\Sigma\left(\mathcal{F}_{c}^{\prime}\right)$ meeting $\stackrel{\circ}{c}$. The dimension of $\sigma^{\prime}$ is $n-\operatorname{dim}\left(c^{\prime}\right)$. The cell $\sigma^{\prime}$ dual to $c^{\prime}$ is a face of the cell $\sigma^{\prime \prime}$ dual to $c^{\prime \prime}$ if and only if $c^{\prime \prime} \prec c^{\prime}$. Notice that this cell complex is of dimension at most $n-1$. It need not be of this dimension, nor even be of homogeneous dimension. We denote by $c_{\sigma}$ the cone in $\mathcal{F}_{c}^{\prime}$ dual to the cell $\sigma \in \Sigma\left(\mathcal{F}_{c}^{\prime}\right)$.

LEMma 2.4. The pre-image $\rho^{-1}\left(\mathcal{O}_{c}\right)$ is given as

$$
\rho^{-1}\left(\mathcal{O}_{c}\right)=\bigcup_{\sigma \in \Sigma\left(\mathcal{F}_{c}^{\prime}\right)} \mathcal{O}_{c_{\sigma}} .
$$

This bijective correspondence between the $T_{N}$-orbits in $\rho^{-1}\left(\mathcal{O}_{c}\right)$ and the cells of $\Sigma\left(\mathcal{F}_{c}^{\prime}\right)$ identifies the face relationship in $\Sigma\left(\mathcal{F}_{c}^{\prime}\right)$ with adherence relationship of orbits.

Proof. Let $c^{\prime}$ be a cone in $\mathcal{F}_{c}^{\prime}$. Then $\rho\left(\mathcal{O}_{c^{\prime}}\right)=\mathcal{O}_{c}$ if and only if every $m \in M \cap \check{c}$ that is not identically zero on $c$ is not identically zero on $c^{\prime}$. Since $c^{\prime}$ contains a relative interior point of $c$ and since $m \geq 0$ on $c$, this is clear. The fact that $\mathcal{O}_{c_{1}}$ is contained in the closure of $\mathcal{O}_{c_{2}}$ if and only if $\left(c_{1}\right)_{\sigma}$ is a face of $\left(c_{2}\right)_{\sigma}$ is clear from Equation (2.2).

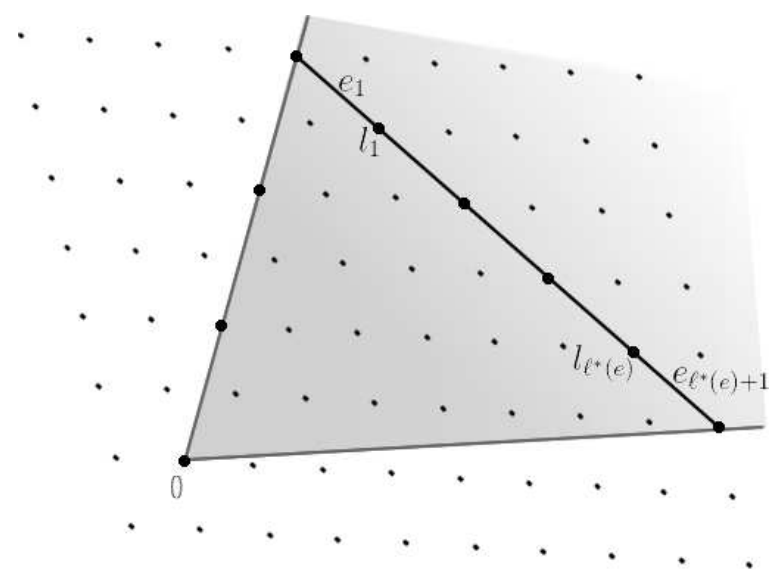

Figure 1. Example 2.5

EXAMPLE 2.5. Let $c$ be the cone on an edge $e$ with vertices indecomposable elements of $N$. Set $k(e)=\ell^{*}(e)+1$. Then $V(e) \simeq \mathbb{C}^{2} /(\mathbb{Z} / k(e) \mathbb{Z})$. The action is given by the primitive $k(e)^{t h}$ root of unity $\zeta$ acting by $\zeta\left(z_{1}, z_{2}\right)=\left(\zeta z_{1}, \zeta^{-1} z_{2}\right)$. In particular, the space $V(e)$ is homeomorphic to $c(L(k(e),-1))$, the cone over the Lens space $L(k(e),-1)$. Consider the subdivision $\mathcal{F}_{e}$ of $e$ given by the decomposition

$$
e=e_{1} \cup \ldots \cup e_{\ell^{*}(e)+1}
$$


with $l_{i}=e_{i} \cap e_{i+1}$ for $i=1, \ldots, \ell^{*}(e)$ the points $N \cap \stackrel{\circ}{e}$. Then $\mathbb{P}_{\mathcal{F}_{e}^{\prime}}$ is smooth and the morphism $\rho: \mathbb{P}_{\mathcal{F}_{e}^{\prime}} \rightarrow V(e)$ resolves the singularity at $0_{e}$ into an $A_{\ell^{*}(e)}$ configuration of rational curves - a chain of $\ell^{*}(e)$ rational curves.

Lemma 2.6. Suppose that the $\mathbb{R}$-span of $c$ is $N$, so that we have the unique fixed point $0_{c}$ of the $T_{N}$-action on $U(c, N)$. Then the fiber $\rho^{-1}\left(0_{c}\right)$ is a compact variety, and $\mathbb{P}_{\mathcal{F}_{c}^{\prime}}$ deformation retracts to $\rho^{-1}\left(0_{c}\right)$.

Proof. Let $\mathbb{R}^{+} \times U(c, N)$ be the action coming from the cone contraction given

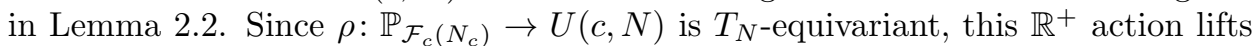
to $\mathbb{P}_{\mathcal{F}^{\prime}}$. This defines a deformation of $\mathbb{P}_{\mathcal{F}_{c}^{\prime}}$ into an arbitrarily small neighborhood of $\rho^{-1}\left(0_{c}\right)$. Of course, a sufficiently small neighborhood of $\rho^{-1}\left(0_{c}\right)$ deformation retracts onto $\rho^{-1}\left(0_{c}\right)$. Compactness of the fiber follows from the properness of $\rho$ (see [Ful] $\S 2.4]$ ).

The refinement $\mathcal{F}_{c}^{\prime}$ of $\mathcal{F}_{c}$ determines a fan denoted $\mathcal{F}_{c}^{\prime}\left(N_{c}\right)$ in $\left(N_{c}\right)_{\mathbb{R}}$. As above we have $\rho: \mathbb{P}_{\mathcal{F}_{c}^{\prime}\left(N_{c}\right)} \rightarrow V(c)$ with $\mathbb{P}_{\mathcal{F}_{c}^{\prime}\left(N_{c}\right)}$ deformation retracting to the compact variety $\rho^{-1}\left(0_{c}\right)$.

CoROllaRY 2.7. $\mathbb{P}_{\mathcal{F}_{c}^{\prime}}$ is a locally trivial fiber bundle over $T_{N} / T_{N_{c}}$ with fiber $\mathbb{P}_{\mathcal{F}_{c}^{\prime}\left(N_{c}\right)}$. The fibers $\rho^{-1}\left(\mathcal{O}_{c}\right) \subset \mathbb{P}_{\mathcal{F}_{c}^{\prime}}$ and $\rho^{-1}\left(0_{c}\right) \subset \mathbb{P}_{\mathcal{F}_{c}^{\prime}\left(N_{c}\right)}$ are related by $\rho^{-1}\left(\mathcal{O}_{c}\right) \cong$ $\rho^{-1}\left(0_{c}\right) \times T_{N} / T_{N_{c}}$.

Proof. Use the fact that $\rho: \mathbb{P}_{\mathcal{F}_{c}^{\prime}} \rightarrow U(c, N)$ is $T_{N}$-equivariant and the fibration structure in Equation 2.1

2.2.2. Refinements and induced maps: The projective case. One class of especially nice lattice polytopes are reflexive polytopes: Given a polytope $\Delta \subset N_{\mathbb{R}}$, define the polar polytope

$$
\Delta^{\circ}=\left\{v \in M_{\mathbb{R}} \mid\langle x, v\rangle \geq-1, \text { for all } x \in \Delta\right\} \subset M_{\mathbb{R}} .
$$

A lattice polytope $\Delta$ is said to be reflexive if $\Delta^{\circ}$ is a lattice polytope in $M_{\mathbb{R}}$.

DeFinition 2.8. Let $\Delta$ be a reflexive polytope. Let $\mathcal{T}$ be a triangulation which is a rectilinear subdivision of $\partial \Delta$, with set of vertices equal to $N \cap \partial \Delta$. The cones of the simplices of $\mathcal{T}$ form a refinement $\mathcal{F}(\mathcal{T})$ of $\mathcal{F}(\Delta)$. Such refinements are called maximal projective subdivisions. We denote by $V(\mathcal{T})$ the set of vertices of the triangulation $\mathcal{T}$, i.e., $V(\mathcal{T})=N \cap \partial \Delta$.

Lemma 2.9. Let $\Delta$ be a reflexive polytope. Let $\mathcal{F}^{\prime}$ be a maximal projective subdivision of $\mathcal{F}(\Delta)$. Let $D_{\infty} \subset \mathbb{P}_{\mathcal{F}^{\prime}}$ be the Weil divisor $\cup_{v \in V(\mathcal{T})} \overline{\mathcal{O}_{v}}$, called the divisor at infinity. For any simplex $\delta$ of $\mathcal{T}$ of dimension $\leq 2, U(\delta, N)$ is smooth and $D_{\infty} \cap U(\delta, N)$ is an anti-canonical divisor in $U(\delta, N)$ and is a divisor with normal crossings in $U(\delta, N)$.

Proof. By reflexivity of $\Delta$, the vertices $\left\{v_{1}, \ldots, v_{r}\right\}$ of any simplex $\delta$ of $\mathcal{F}^{\prime}$ of dimension $\leq 2$ form part of a basis for $N\left[\mathbf{C K}\right.$ Corollary A.2.3]; let $\left\{v_{1}, \ldots, v_{r}, e_{r+1}, \ldots, e_{n}\right\}$ be a basis for $N$. Use this basis to split $N=A \oplus B$ with $v_{1}, \ldots, v_{r}$ being a basis for $A$ and $e_{r+1}, \ldots, e_{n}$ a basis for $B$. Let $A_{+}^{*}$ denote the "positive $2^{r}$-ant" in the dual space $A^{*}$ with respect to the dual basis. Then

$$
M \cap \check{c}=A_{+}^{*} \times B^{*}
$$

so that

$$
U(\delta, N)=\operatorname{Spec} \mathbb{C}[M \cap \check{c}]=\mathbb{C}_{A} \times T_{B}
$$


where $\mathbb{C}_{A}=\mathbb{C} \otimes_{\mathbb{Z}} A$ and

$$
D_{\infty} \cap U(\delta, N)=\cup_{i=1}^{r} H_{i} \times T_{B}
$$

where $H_{i} \subset \mathbb{C}_{A}$ is the $i$ th coordinate hyperplane. Let $t_{i}: T_{N} \rightarrow \mathbb{C}^{*}$ be the coordinate functions for this basis. Then

$$
\frac{d t_{1}}{t_{1}} \wedge \cdots \wedge \frac{d t_{n}}{t_{n}}
$$

is a holomorphic differential $n$-form on $T_{N}$ with simple poles along $D_{\infty}$ Ful $\left.\S 4.3\right]$, proving that $D_{\infty}$ is anti-canonical.

In order to capture all of these properties we make the following definition.

Definition 2.10. Let $\Sigma$ be a finite cell complex, not necessarily of homogeneous dimension. Let $\mathcal{S}$ be a compact complex algebraic variety. We say that $\Sigma$ is a combinatorial model for $\mathcal{S}$ if the following hold:

- For each $i$-cell $c$ of $\Sigma$ there is a locally closed, irreducible algebraic subset $S_{c}$ of $\mathcal{S}$ and $S_{c}$ is isomorphic to a complex torus of dimension $i$.

- $\mathcal{S}=\cup_{c} S_{c}$.

- For $c \neq c^{\prime}, S_{c} \cap S_{c^{\prime}}=\emptyset$.

- For every cell $c$ of $\Sigma$ the closure $\bar{S}_{c}$ of $S_{c}$ is isomorphic to a smooth variety and

$$
\bar{S}_{c}=\bigcup_{\left\{c^{\prime} \prec c\right\}} S_{c^{\prime}} .
$$

- For every cell $c$ of $\Sigma$ the subset $\cup_{\left\{c^{\prime} \prec c \mid c^{\prime} \neq c\right\}} S_{c^{\prime}}$ in $S_{c}$ is a smooth divisor with normal crossings in $\bar{S}_{c}$. It is an anti-canonical divisor for $\bar{S}_{c}$.
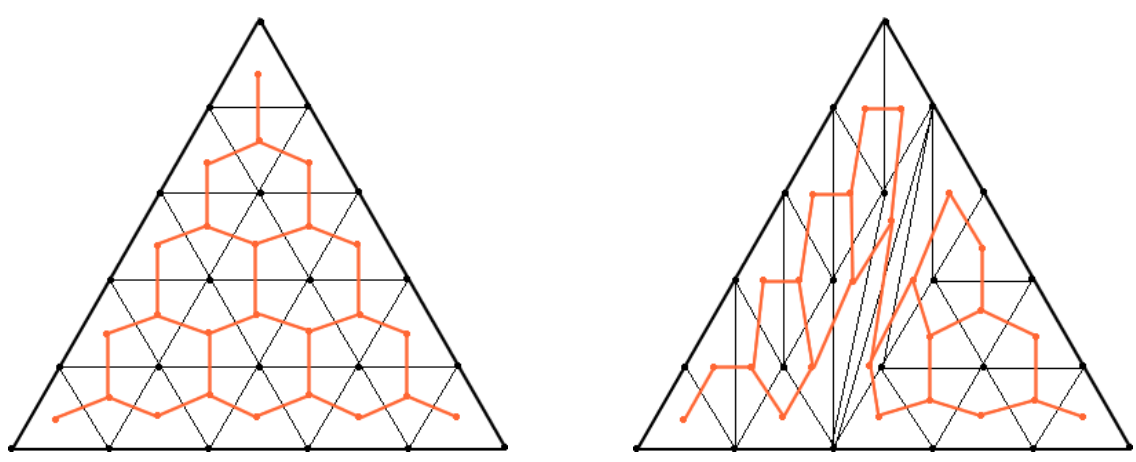

Figure 2. Examples of triangulated two-faces with dual cell complexes

Proposition 2.11. Let $\Delta$ be a reflexive polytope, let $\mathcal{F}^{\prime}$ be a maximal projective subdivision of $\mathcal{F}(\Delta)$, and let $f$ be a face of $\partial \Delta$. Let $\rho: \mathbb{P}_{\mathcal{F}_{f}^{\prime}(N)} \rightarrow U(f, N)$ be the map given in Lemma 2.3 and let $\rho_{f}$ be the restriction of $\rho$ to $\mathbb{P}_{\mathcal{F}_{f}^{\prime}\left(N_{f}\right)} \subset \mathbb{P}_{\mathcal{F}_{f}^{\prime}(N)}$. Denote by $\Sigma\left(\mathcal{F}_{f}^{\prime}\right)$ the cell complex $\Sigma\left(\mathcal{F}_{c(f)}^{\prime}\right)$. Then $\Sigma\left(\mathcal{F}_{f}^{\prime}\right)$ is a combinatorial model for $\mathcal{S}(f)=\rho^{-1}\left(0_{f}\right) \subset \mathbb{P}_{\mathcal{F}_{f}^{\prime}\left(N_{f}\right)}$. Furthermore, under the map $\rho: \mathbb{P}_{\mathcal{F}_{f}^{\prime}(N)} \rightarrow U(f, N)$ the preimage of the closed orbit $\mathcal{O}_{f}$ is isomorphic to $\mathcal{S}(f) \times T_{N} / T_{N_{f}}$. 
Proof. By Lemma 2.4 $\rho^{-1}\left(0_{f}\right)$ is the union of all the $T_{N_{c}}$-orbits in $\mathcal{F}_{f}^{\prime}\left(N_{f}\right)$ corresponding to simplices of $\mathcal{F}^{\prime}$ meeting $\stackrel{\circ}{f}$. The dimension of the orbit corresponding to a simplex $\delta$ is the codimension of $\delta$ in $f$. Hence there is a bijective correspondence between the $i$-cells of $\Sigma\left(\mathcal{F}^{\prime}\right)$ and the $i$-dimensional $T_{N_{f}}$-orbits in $\rho^{-1}\left(0_{f}\right)$. By Equation (2.2), under this correspondence the face relation becomes the adherence relation. This establishes the first four conditions in the definition. The fifth is immediate from Lemma 2.9.

The last statement is immediate from Corollary 2.7

2.3. Lefschetz theorems and arithmetic genus of Calabi-Yau manifolds in toric varieties. Suppose that $N$ is a lattice of rank 4 and $\Delta \subset N_{\mathbb{R}}$ is a reflexive polytope with $\mathbb{P}_{\Delta}$ as associated toric variety and $D_{\infty}$ the divisor at infinity. Since $\mathcal{O}\left(D_{\infty}\right)$ is semi-ample [CK Lemma 4.1.2], the sections of this bundle define a projective embedding

$$
\iota: \mathbb{P}_{\Delta} \hookrightarrow \mathbb{P}\left(H^{0}\left(\mathbb{P}_{\Delta}, \mathcal{O}\left(D_{\infty}\right)\right)^{\vee}\right) .
$$

Let $\mathcal{T}$ be a maximal triangulation of $\Delta$, i.e., one whose set of vertices is $N \cap \partial \Delta$. Taking the cones over the simplices of $\mathcal{T}$ defines a maximal projective subdivision $\mathcal{F}^{\prime}=\mathcal{F}^{\prime}(\mathcal{T})$ of $\mathcal{F}(\Delta)$. There are the associated projective variety $\mathbb{P}_{\mathcal{F}^{\prime}}$ and the $T_{N}$-equivariant morphism

$$
\rho: \mathbb{P}_{\mathcal{F}^{\prime}} \rightarrow \mathbb{P}_{\Delta} .
$$

Recall that for each face $\gamma$ of $\partial \Delta, \mathcal{F}_{\gamma}^{\prime}\left(N_{\gamma}\right)$ is the sub-fan of $\mathcal{F}^{\prime}$ consisting of all cones in $\mathcal{F}^{\prime}$ contained in $c(\gamma)$. According to [CK Lemma 4.1.2 and Corollary A.2.3] for every face $\gamma$ of $\partial \Delta$ of positive codimension, $\mathbb{P}_{\mathcal{F}_{\gamma}^{\prime}\left(N_{\gamma}\right)}$ is smooth.

Let $Y \subset \mathbb{P}_{\Delta}$ be the intersection of $\mathbb{P}_{\Delta}$ with a generic hyperplane in $\mathbb{P}\left(H^{0}\left(\mathbb{P}_{\Delta}, \mathcal{O}\left(D_{\infty}\right)\right)^{\vee}\right)$. Then $Y$ is a generic anticanonical divisor in $\mathbb{P}_{\Delta}$ transverse to all $T_{N}$-orbits. We set $\widetilde{Y}=\rho^{-1}(Y) \subset \mathbb{P}_{\mathcal{F}^{\prime}}$. It is also anti-canonical and, as was the case with $\mathbb{P}_{\mathcal{F}_{\gamma}^{\prime}\left(N_{\gamma}\right)}$ above, $\widetilde{Y}$ is a smooth Calabi-Yau threefold. For any lattice point $\ell \in N \cap \partial \Delta$, there is a toric divisor $\widetilde{\mathcal{O}}_{\ell} \subset \mathbb{P}_{\mathcal{F}^{\prime}}$. The only singularities of its closure in $\mathbb{P}_{\mathcal{F}^{\prime}}$ occur at fixed points of the $T_{N}$-action. Thus, the intersection of $\widetilde{Y}$ with the closure of $\widetilde{\mathcal{O}}_{\ell}$ is a smooth divisor in $\tilde{Y}$. We denote it by $R(\ell)$. Its image in $Y$ is the intersection of $Y$ with the closure of the orbit corresponding to the unique open face of $\partial \Delta$ containing $\ell$.

Let us turn to the analogue for $\Delta$ of dimension $n>4$ and complete intersections. First we need to recall the notion of Batyrev and Borisov of NEF partitions defining complete intersections. Let $\Delta \subset N_{\mathbb{R}}$ be a reflexive polytope. Let $V(\Delta)=V_{1} \amalg \cdots \amalg V_{k}$ be a partition of the vertices of $\Delta$. According to $\left.\mathbf{B B} \S 4\right]$ this is a NEF partition if there are functions $\varphi_{i}: N_{\mathbb{R}} \rightarrow \mathbb{R}, 1 \leq i \leq k$, with the following properties:

(1) $\varphi_{i}$ is piecewise linear and linear on the cone on each face of $\Delta$.

(2)

$$
\varphi_{i}(v)=\left\{\begin{array}{ll}
0 & \text { if } v \notin V_{i} \\
-1 & \text { if } v \in V_{i}
\end{array} .\right.
$$

(3) $\varphi_{i}$ is a concave function; i.e., $\varphi_{i}(t a+(1-t) b) \geq t \varphi_{i}(a)+(1-t) \varphi_{i}(b)$. 
(4) For each $i \leq k$ and each maximal dimensional face $f$ of $\partial \Delta$ there is an $m_{i, f} \in M$ such that

$$
\left.\varphi_{i}\right|_{c(f)}=\left\langle m_{i, f}, \cdot\right\rangle \text {. }
$$

Set $\nabla_{i}^{\circ} \subset M_{\mathbb{R}}$ equal to the convex hull of the $\left\{m_{i, f}\right\}$ as $f$ ranges over the maximal dimensional faces of $\partial \Delta$. Then

$$
\varphi_{i}(x)=\min _{y \in \nabla_{i}^{\circ}}\langle y, x\rangle .
$$

Let $D_{i}=\sum_{v \in V_{i}} \overline{\mathcal{O}}_{v}$. Clearly, $D_{\infty}=D_{1}+\cdots+D_{k}$ is anti-canonical. The divisor $D_{i}$ is ample if and only if the function $\varphi_{i}$ is strictly convex on $\partial \Delta$, see Ful, p. 70]. Now let us suppose that we have a NEF partition $V(\Delta)=V_{1} \amalg \cdots \amalg V_{k}$ with the property that each of the corresponding divisors $D_{i}$ is ample. This condition implies that $\nabla_{i}^{\circ}$ is combinatorially dual to $\Delta$.

We need the following elementary lemma from $\mathbf{B B}$ Proposition 6.3].

LEMMA 2.12. Suppose that $\gamma$ is a face of $\partial \Delta$ and suppose that the relative interior of $\gamma$ contains a lattice point. Then there is an $i \leq k$ such that all vertices of $\gamma$ belong to the same $V_{i}$. We denote by $i(\gamma)$ this index.

Suppose that the relative interior of $\gamma$ contains a lattice point. Then all the vertices of $\gamma$ are contained in $V_{i(\gamma)}$, and hence for every $j \neq i(\gamma)$ we define the dual of $\gamma$ in $\nabla_{j}^{\circ}$, denoted $\gamma_{j}^{\vee}$, to be the face of $\nabla_{j}^{\circ}$ that evaluates 0 on $\gamma$. Analogously, we define $\gamma_{i(\gamma)}^{\vee}$ to be the face of $\nabla_{i(\gamma)}^{\circ}$ that evaluates -1 on $\gamma$. Since $D_{i}$ is ample, $\gamma_{i}^{\vee}$ is the face of $\nabla_{i}^{\circ}$ dual to the face $\gamma$ of $\partial \Delta$ under the combinatorial duality. In particular, for each face $\gamma$ of $\partial \Delta$ the dimension of $\gamma_{i}^{\vee}$ is $n-1-\operatorname{dim}(\gamma)$.

2.3.1. Lefschetz theorems. We need a generalization of the classical Lefschetz theorem, a generalization established by Bernstein, Danilov and Khovanskii (DK Theorem 6.4]). Consider the generic affine complete intersection $Y_{0} \subset \mathbb{T}^{n}=\left(\mathbb{C}^{*}\right)^{n}$ of dimension $r=n-k$.

THEOREM 2.13. The compactly supported cohomology $H_{c}^{i}\left(Y_{0}\right)=0$ for $i<r$. Furthermore, if all the Newton polyhedra $\Delta_{1}, \ldots, \Delta_{k}$ have dimension $n$, then the Gysin homomorphism $H_{c}^{i}\left(Y_{0}\right) \rightarrow H_{c}^{i+2 k}\left(\mathbb{T}^{n}\right)$ is an isomorphism for $i>r$ and surjective for $i=r$.

The first statement is classical, given that $Y_{0}$ is a smooth affine variety of dimension $r$.

Applying Poincaré duality for noncompact manifolds yields:

COROLlaRY 2.14. Under the hypotheses of Theorem 2.13 the map in homology induced by the inclusion,

$$
H_{i}\left(Y_{0}\right) \rightarrow H_{i}\left(\mathbb{T}^{n}\right)
$$

is an isomorphism for $i$ less than the complex codimension $k$ of $Y_{0}$ and surjective for $i=k$.

It will be important to have these results not only for the open torus-orbit in $P_{\Delta}$ but also for orbits of smaller dimension. In the hypersurface case, let $\omega$ be a generic section of $\mathcal{O}\left(D_{\infty}\right)$. Consider a face $\gamma$ of $\Delta$. The restriction of $\omega$ to the orbit $\mathcal{O}_{\gamma}$ has convex hull an affine translate of $\gamma^{\vee} \subset \Delta^{\circ}$ and hence the dimension of this convex hull is equal to the dimension of the orbit $\mathcal{O}_{\gamma}$. Thus, the Lefschetz theorem applies to $Y \cap \mathcal{O}_{\gamma} \rightarrow \mathcal{O}_{\gamma}$. But notice that the dimension of $Y \cap \mathcal{O}_{\gamma}$ is smaller than 
$r$, and the comparison of homologies only goes up to the middle (real) dimension of this variety.

In the complete intersection case, the necessary dimension hypothesis does not automatically restrict to the faces. For this we need the $D_{i}$ to be ample.

Lemma 2.15. Let $\Delta \subset N_{\mathbb{R}}$ be a reflexive polytope and let $V(\Delta)=V_{1} \amalg \cdots \amalg V_{k}$ be a NEF partition. Suppose that each of the divisors $D_{i}$ associated to the $V_{i}$ are ample. Let $\omega_{i}$ be a generic section of $D_{i}$. Then for each face $\gamma$ of $\partial \Delta$, the convex hull of the support of $\left.\omega_{i}\right|_{\gamma}$ has dimension equal to the dimension of $\mathcal{O}_{\gamma}$.

Proof. The convex hull of the support of $\left.\omega_{i}\right|_{\gamma}$ is an affine translate of $\gamma_{i}^{\vee} \subset \nabla_{i}^{\circ}$. Since $D_{i}$ are ample, the $\nabla_{i}^{\circ}$ are combinatorially dual to $\Delta$, and hence the faces $\gamma_{i}^{\vee}$ have dimension $n-1-\operatorname{dim}(\gamma)$, which is exactly the dimension of $\mathcal{O}_{\gamma}$.

Now applying Corollary 2.14 gives the following:

Proposition 2.16. Let $\Delta \subset N_{\mathbb{R}}$ be a reflexive polytope. Let $Y \subset P_{\Delta}$ be an $r$-dimensional variety, either a generic anti-canonical hypersurface or a generic complete intersection associated to a NEF partition $D_{\infty}=D_{1}+\cdots+D_{k}$, with the $D_{i}$ being ample. Then for each face $\gamma$ of $\Delta$ the inclusion $Y \cap \mathcal{O}_{\gamma} \rightarrow \mathcal{O}_{\gamma}$ induces an isomorphism on homology below dimension $(r-\operatorname{codim}(\gamma))$ and induces a surjection on homology in that dimension.

2.3.2. Arithmetic genus. We begin by recalling a result of Khovanskii ( Kho Theorem 1]) in the case of hypersurfaces in a torus.

Proposition 2.17. Let $\mathbb{C}^{*} \otimes N$ be a torus, and let $\omega \in \mathbb{C}[\mathbb{T}]$ be a non-degenerate regular function on $\mathbb{C}^{*} \otimes N$, non-degenerate in the sense that $Y=\omega^{-1}(0)$ is a smooth hypersurface. Let $\Delta^{\prime} \subset M_{\mathbb{R}}$ be the convex hull of the support of $\omega$. Then the arithmetic genus $\chi(Y)$ is given by

$$
\chi(Y)=1-B\left(\Delta^{\prime}\right)
$$

where

$$
B\left(\Delta^{\prime}\right)=(-1)^{\operatorname{dim}\left(\Delta^{\prime}\right)} \#\left(\Delta^{\prime} \cap M\right) .
$$

We apply this to our context. First, let us also suppose that $N$ is of rank 4 and that $\Delta \subset N \otimes \mathbb{R}$ is reflexive. Let $\gamma$ be a face of $\partial \Delta$ and denote by $\gamma^{\vee}$ the dual face in $\Delta^{\circ}$ consisting of all $x \in \Delta^{\circ}$ with the property that $\langle x, \gamma\rangle=-1$. Under the mapping $\mathbb{P}_{\Delta} \rightarrow \mathbb{P}\left(H^{0}\left(\mathbb{P}_{\Delta}, \mathcal{O}\left(D_{\infty}\right)\right)^{\vee}\right)=\mathbb{P}\left(\mathbb{C}^{M \cap \Delta^{\circ}}\right)$ the image of the orbit $\mathcal{O}_{\gamma}$ is contained in the subprojective space determined by the vanishing of all $m \in \Delta^{\circ} \backslash \gamma^{\vee}$. Thus, the supporting polytope for the restriction of a generic section

$$
\omega=\sum_{m \in M \cap \Delta^{\circ}} a_{m} \chi^{m}
$$

of $\mathcal{O}\left(D_{\infty}\right)$ to $\mathcal{O}_{\gamma}$ is the convex hull of $M \cap \gamma^{\vee}$. Since the vertices of $\gamma^{\vee}$ are contained in $M$, the supporting polytope is $\gamma^{\vee}$. Applying the Khovanskii result cited above to $\mathcal{O}_{\gamma}$ gives the following:

Corollary 2.18. Let $N$ be a lattice of rank $4, \Delta \subset N_{\mathbb{R}}$ a reflexive polytope, $\omega$ a generic section of $\mathcal{O}\left(D_{\infty}\right)$, and $Y \subset \mathbb{P}_{\Delta}$ the zero locus of this section. Then for any edge $e$ of $\Delta$ the intersection $Y \cap \mathcal{O}_{e}$ is a (non-compact) riemann surface of genus $\ell^{*}\left(e^{\vee}\right)$. For any two-face $f$ of $\Delta$ the intersection $Y \cap \mathcal{O}_{f}$ consists of $1+\ell^{*}\left(f^{\vee}\right)$ points. 
Let's now generalize this to the complete intersection case.

Theorem 1 of Kho also implies the following.

Proposition 2.19. The arithmetic genus $\chi(Y)$ of the variety $Y$ defined in $\left(\mathbb{C}^{*}\right)^{n}$ by a nondegenerate system of equations $\omega_{1}=\ldots=\omega_{k}=0$ with Newton polyhedra $\Delta_{1}^{\prime}, \ldots, \Delta_{k}^{\prime}$ is given by

$$
\chi(Y)=1-\sum_{i} B\left(\Delta_{i}^{\prime}\right)+\sum_{i<j} B\left(\Delta_{i}^{\prime}+\Delta_{j}^{\prime}\right)-\ldots+(-1)^{k} B\left(\Delta_{1}^{\prime}+\ldots+\Delta_{k}^{\prime}\right),
$$

where

$$
B\left(\Delta^{\prime}\right)=(-1)^{\operatorname{dim}\left(\Delta^{\prime}\right)} \#\left(\Delta^{\prime} \cap M\right) .
$$

Applying this result to the various faces gives:

Corollary 2.20. Let $\Delta \subset N_{\mathbb{R}}$ be a reflexive polytope, where $\operatorname{dim} N_{\mathbb{R}}=n=$ $k+3$. Let

$$
V(\Delta)=\coprod_{i \in I} V_{i}
$$

where $I=\{1, \ldots, k\}$, be a NEF partition with the associated divisors $D_{i}$ being ample. Let $Y \subset \mathbb{P}_{\Delta}$ be a generic complete intersection of sections of the $\mathcal{O}\left(D_{i}\right)$. Then:

(1) For any 2-face $f$ of $\partial \Delta$ whose relative interior contains a lattice point,

$$
\#\left(Y \cap \mathcal{O}_{f}\right)=1+\sum_{J \subset I ; J \neq \emptyset}(-1)^{3-|J|} \ell^{*}\left(\sum_{j \in J} f_{j}^{\vee}\right) .
$$

(2) For an edge e of $\partial \Delta$ containing an interior lattice point, the curve $Y \cap \mathcal{O}_{e}$ has

$$
\chi\left(Y \cap \mathcal{O}_{e}\right)=1-\sum_{J \subset I ; J \neq \emptyset}(-1)^{r-|J|} \ell^{*}\left(\sum_{j \in J} e_{j}^{\vee}\right) .
$$

Notice that when $k=1$, this formula specializes to the one given in Corollary 2.18

2.4. First computations of homology groups of Calabi-Yau threefolds. Throughout this section we suppose that $\Delta$ is a reflexive polytope and that either the dimension of $\Delta$ is 4 and $Y$ is the vanishing locus of a generic section od $\mathcal{O}\left(D_{\infty}\right)$, or that the dimension of $\Delta$ is $n=k+3$ and we have a NEF partition $V(\Delta)=V_{1} \amalg \cdots \amalg V_{k}$ with the corresponding divisors $D_{1}, \ldots, D_{k}$ being ample with $Y$ being the complete intersection of the zero loci of generic sections of the $D_{i}$. In either case $Y$ is a (possibly singular) Calabi-Yau three-fold. Since $\widetilde{Y}=\rho^{-1}(Y) \subset \mathbb{P}_{\mathcal{T}}$ is smooth, it is easy to compute $H_{1}(\widetilde{Y})$ and $H_{2}(\widetilde{Y})$ given the Lefschetz theory described above.

Let $\widetilde{Y}_{0}$ be the intersection of $\widetilde{Y}$ with the open $T_{N}$-orbit in $\mathbb{P}_{\mathcal{T}}$. By the Lefschetz theorem (Section 2.3.1) we have

$$
\begin{aligned}
& H_{0}\left(\widetilde{Y}_{0}\right)=H_{0}\left(Y_{0}\right)=\mathbb{Z} \\
& H_{1}\left(\widetilde{Y}_{0}\right)=H_{1}\left(Y_{0}\right)=N \\
& H_{2}\left(\widetilde{Y}_{0}\right)=H_{2}\left(Y_{0}\right)=\wedge^{2} N .
\end{aligned}
$$


We set $\widetilde{W} \subset \widetilde{Y}$ equal to the union of $Y_{0}$ and all the intersections of $\widetilde{Y}$ with the codimension-one torus orbits in $\mathbb{P}_{\mathcal{T}}$. Of course, these orbits are indexed by the vertices of $\mathcal{T}$, i.e., by $N \cap \partial \Delta$. Thus, the pair $\left(\widetilde{W}, \widetilde{Y}_{0}\right)$ is excisively equivalent to

$$
\coprod_{\ell \in \partial \Delta \cap N}\left(\tilde{Y} \cap \widetilde{\mathcal{O}}_{\ell}\right) \times\left(D^{2}, S^{1}\right) .
$$

Let's use the Lefschetz theory to analyze $\widetilde{Y} \cap \widetilde{\mathcal{O}}_{\ell}$.

LEMma 2.21. (1) If $\ell \in V(\partial \Delta)$, then $\tilde{Y} \cap \widetilde{\mathcal{O}}_{\ell}=Y \cap \mathcal{O}_{\ell}$. Furthermore, $H_{0}\left(\widetilde{Y} \cap \widetilde{\mathcal{O}}_{\ell}\right)=\mathbb{Z}$ and the inclusion $\widetilde{Y} \cap \widetilde{\mathcal{O}}_{\ell} \rightarrow \widetilde{\mathcal{O}}_{\ell}$ induces an isomorphism $H_{1}\left(\widetilde{Y} \cap \widetilde{\mathcal{O}}_{\ell}\right) \rightarrow H_{1}\left(\widetilde{\mathcal{O}}_{\ell}\right)$.

(2) If $\ell$ is contained in the interior of an edge e of $\Delta$, then $\widetilde{Y} \cap \widetilde{\mathcal{O}}_{\ell}$ is a torus-fibration over $Y \cap \mathcal{O}_{e}$ with (one-dimensional) fiber

$$
\frac{N \cap \mathbb{R}-\operatorname{span}(e)}{N \cap \mathbb{R}-\operatorname{span}(\ell)} \otimes \mathbb{C}^{*}
$$

Furthermore, $\tilde{Y} \cap \widetilde{\mathcal{O}}_{\ell}$ is connected and the inclusion $\tilde{Y} \cap \widetilde{\mathcal{O}}_{\ell} \rightarrow \widetilde{\mathcal{O}}_{\ell}$ induces a surjection on $H_{1}$.

(3) If $\ell$ is contained in the interior of a two-face $f$ of $\Delta$, then $\widetilde{Y} \cap \widetilde{\mathcal{O}}_{\ell}$ is a torus fibration over $Y \cap \mathcal{O}_{f}$, which is a finite, non-empty set of points. The fiber is identified with the complex two-torus

$$
\frac{N \cap \mathbb{R}-\operatorname{span}(f)}{N \cap \mathbb{R}-\operatorname{span}(\ell)} \otimes \mathbb{C}^{*} .
$$

In particular, each component of $\widetilde{Y} \cap \widetilde{\mathcal{O}}_{\ell}$ has first homology isomorphic to

$$
\frac{N \cap \mathbb{R}-\operatorname{span}(f)}{N \cap \mathbb{R}-\operatorname{span}(\ell)}
$$

inside $H_{1}\left(\widetilde{\mathcal{O}}_{\ell}\right)=N /\langle\ell\rangle$.

(4) If $\ell$ is contained in the interior of a three-face of $\partial \Delta$, then $\widetilde{Y} \cap \widetilde{\mathcal{O}}_{\ell}=\emptyset$.

PROOF. If $\ell$ is contained in the interior of a face $\gamma$ of $\Delta$, then the restriction of the map $\rho$ to a map $\widetilde{\mathcal{O}}_{\ell} \rightarrow \mathcal{O}_{\gamma}$ is the natural projection

$$
\frac{N}{\mathbb{Z}\langle\ell\rangle} \otimes \mathbb{C}^{*} \rightarrow \frac{N}{\mathbb{R}-\operatorname{span}(\gamma)} \otimes \mathbb{C}^{*}
$$

Since $Y \subset X$ is generic, $\tilde{Y} \cap \mathcal{O}_{\gamma}$ is a fibration with fiber

$$
\frac{\mathbb{R}-\operatorname{span}(\gamma)}{\mathbb{Z}\langle\ell\rangle} \otimes \mathbb{C}^{*}
$$

When $\ell$ is a vertex of $\partial \Delta, \widetilde{Y} \cap \widetilde{\mathcal{O}}_{\ell}=Y \cap \mathcal{O}_{\ell}$ and the homology statements in part (1) are verified in Proposition 2.16

Let us suppose that $\ell$ is contained in the interior of an edge $e$ of $\Delta$. Then by Proposition [2.16] we have $Y \cap \mathcal{O}_{e}$ is connected and $H_{1}\left(Y \cap \mathcal{O}_{e}\right) \rightarrow H_{1}\left(\mathcal{O}_{e}\right)$ is surjective. Since $\widetilde{Y} \cap \widetilde{\mathcal{O}}_{\ell} \rightarrow \widetilde{\mathcal{O}}_{\ell}$ is a $\mathbb{C}^{*}$-bundle over the inclusion $Y \cap \mathcal{O}_{e} \rightarrow \mathcal{O}_{e}$, the result follows in this case.

Now suppose that $\ell$ is contained in the interior of a two-face $f$. By Proposition 2.16 $Y \cap \mathcal{O}_{f}$ is a finite, non-empty, set of points. The result is then clear in this case. 
Lastly, if $\ell$ is contained in the interior of a three-face $g$, then the generic $Y$ in the given linear system misses the point $\mathcal{O}_{g}$ in $P_{\Delta}$. Hence, the preimage $\tilde{Y}$ misses $\widetilde{\mathcal{O}}_{\ell}$.

Using the long exact sequence of the pair $\left(\widetilde{W}, \widetilde{Y}_{0}\right)$ gives

$\bigoplus_{\ell \in \partial \Delta^{(2)} \cap N} H_{1}\left(\widetilde{\mathcal{O}}_{\ell} \cap \tilde{Y}\right) \stackrel{\oplus_{\ell}(\cdot \wedge \ell)}{\longrightarrow} \wedge^{2} N \rightarrow H_{2}(\widetilde{W}) \rightarrow \bigoplus_{\ell \in \partial \Delta^{(2)} \cap N} H_{0}\left(\widetilde{\mathcal{O}}_{\ell} \cap \tilde{Y}\right) \stackrel{\oplus_{\ell} \cdot \ell}{\rightarrow} N \rightarrow H_{1}(\widetilde{W}) \rightarrow 0$.

Here, the notation $\partial \Delta^{(2)}$ refers to the two-skeleton of $\partial \Delta$. The first map is the direct sum over $\ell$ of the compositions

$$
H_{1}\left(\widetilde{Y} \cap \widetilde{\mathcal{O}}_{\ell}\right) \rightarrow H_{1}\left(\widetilde{\mathcal{O}}_{\ell}\right)=N /\langle\ell\rangle \stackrel{\cdot \wedge \ell}{\rightarrow} \wedge^{2} N
$$

Using this we shall show the following result.

Corollary 2.22. $\quad$ (1) $H_{1}(\widetilde{Y})=N / \operatorname{Span}\left(N \cap \partial \Delta^{(2)}\right)$.

(2) We have an exact sequence:

$$
0 \rightarrow \operatorname{Tor} H_{2}(\tilde{Y}) \rightarrow H_{2}(\tilde{Y}) \rightarrow \operatorname{Ker}\left(\bigoplus_{\ell}(\cdot \ell)\right) \rightarrow 0 .
$$

(3) $\operatorname{Ker}\left(\bigoplus_{\ell}(\cdot \ell)\right)$ is free abelian. In the hypersurface case it is of rank

$$
\operatorname{rank} \operatorname{Ker}\left(\bigoplus_{\ell}(\cdot \ell)\right)=\# V+\sum_{e} \ell^{*}(e)+\sum_{f} \ell^{*}(f)\left(1+\ell^{*}\left(f^{\vee}\right)\right)-4 .
$$

In the complete intersection case (of ample divisors) its rank is

$\operatorname{rank} \operatorname{Ker}\left(\bigoplus_{\ell}(\cdot \ell)\right)=\# V+\sum_{e} \ell^{*}(e)+\sum_{f} \ell^{*}(f)\left(1+\sum_{J \subset I ; J \neq \emptyset}(-1)^{3-|J|} \ell^{*}\left(\sum_{j \in J} f_{j}^{\vee}\right)\right)-n$.

$$
\text { Tor } H_{2}(\tilde{Y})=\wedge^{2} N / \text { Image }\left(\bigoplus_{\ell \in \partial \Delta(1) \cap N} \frac{N}{N \cap \mathbb{R}-\operatorname{span}(\ell)} \wedge \ell \oplus \bigoplus_{f}\left(\bigoplus \frac{N \cap \mathbb{R}-\operatorname{span}(f)}{N \cap \mathbb{R}-\operatorname{span}(\ell)} \wedge \ell\right)\right) \text {. }
$$

Proof. Since the complement of $\widetilde{W}$ in $\widetilde{Y}$ is a union of orbits of complex codimension at least two, and since $\widetilde{Y}$ is smooth, it follows by general position that $H_{i}(\widetilde{W}) \rightarrow H_{i}(\widetilde{Y})$ is an isomorphism for $i \leq 2$. Hence, we work with $H_{i}(\widetilde{W})$ for $i=1,2$.

The first statement is immediate from the long exact sequence of the pair $\left(\widetilde{W}, \widetilde{Y}_{0}\right)$ since $\widetilde{\mathcal{O}}_{\ell} \cap \widetilde{Y}$ is non-empty for every $\ell \in \partial \Delta^{(2)}$. It is easy to see that the first map in this exact sequence has torsion cokernel. Since the fourth term is free abelian, the second statement follows. It also follows that $\operatorname{Ker}\left(\bigoplus_{\ell}(\cdot \ell)\right)$ is free abelian. Let us compute its rank, which is the rank of fourth term minus $n$, the rank of $N$. By the Lefschetz theorem $\widetilde{\mathcal{O}}_{\ell} \cap \widetilde{Y}$ is connected if $\ell$ is contained in the one-skeleton of $\partial \Delta$. By Corollary 2.18 in the hypersurface case $\widetilde{\mathcal{O}} \ell \cap \widetilde{Y}$ has $\left(1+\ell^{*}\left(f^{\vee}\right)\right)$ components if $\ell$ is contained in the relative interior of two-face $f$. By Corollary 2.20 in the complete intersection case if $\ell$ is contained in the relative interior of a two-face then $\widetilde{\mathcal{O}}_{\ell} \cap \widetilde{Y}$ ) has

$$
1+\sum_{J \subset I ; J \neq \emptyset}(-1)^{3-|J|} \ell^{*}\left(\sum_{j \in J} f_{j}^{\vee}\right)
$$


components. In both cases the formula for the rank is immediate. The fourth item is clear from our description of $H_{1}\left(\widetilde{\mathcal{O}}_{\ell} \cap \widetilde{Y}\right)$.

Notice that parts (2) and (3) of this result establish part (1) of Theorem 1.2 and gives the more general formula for the rank of $H_{2}(\widetilde{Y})$ in the complete intersection case.

2.4.1. Representation by divisors. We now establish the results in part (2) of Theorem 1.2

Proposition 2.23. For every $\alpha \in H^{2}(\tilde{Y})$, there exists a integral linear combination of divisors $D=\sum_{j} n_{j} D_{j}$ such that $\alpha$ is Poincaré dual to $D$. In fact, each irreducible component $D_{j}$ in this sum can be taken to be an irreducible component of the intersection of $\widetilde{Y}$ with the closure of a torus-orbit of codimension one in $\widetilde{X}$.

Proof. We have the exact sequence

$$
\bigoplus_{\ell} H^{0}\left(\widetilde{Y} \cap \widetilde{\mathcal{O}}_{\ell}\right) \rightarrow H^{2}(\widetilde{Y}) \rightarrow H^{2}\left(\widetilde{Y}_{0}\right)
$$

The image of the generator of one of the $\mathbb{Z}$-summands in $H^{0}\left(\widetilde{Y} \cap \widetilde{\mathcal{O}}_{\ell}\right)$ maps to the Poincaré dual of the corresponding divisor in $H^{2}(\widetilde{Y})$. Thus, the proposition follows immediately if we can show that the map $H^{2}(\widetilde{Y}) \rightarrow H^{2}\left(\tilde{Y}_{0}\right)$ is trivial. Since $H^{2}\left(\widetilde{Y}_{0}\right)=H^{2}\left(T_{N}\right)$ is torsion free, it suffices to show that the algebraically dual map

$$
H_{2}\left(\widetilde{Y}_{0}\right) \rightarrow H_{2}(\widetilde{Y})
$$

has torsion image. Of course, the inclusion $\widetilde{W} \subset \widetilde{Y}$ induces an isomorphism $H_{2}(\widetilde{W}) \rightarrow H_{2}(\widetilde{Y})$, and hence we need only see that

$$
\bigoplus_{\ell} H_{1}\left(\widetilde{\mathcal{O}}_{\ell} \cap \tilde{Y}\right) \rightarrow \wedge^{2} N=H_{2}\left(\widetilde{Y}_{0}\right)
$$

$\wedge^{2} N=H_{2}\left(\widetilde{Y}_{0}\right) \rightarrow H_{2}(\widetilde{Y})$ has torsion image. This we already observed in the proof of Corollary 2.22

This completes the proof.

Corollary 2.24. The Hodge structure on $H^{2}(\widetilde{Y})$ is of type $(1,1)$.

Corollary 2.25. The mixed Hodge structure of $H^{2}(Y)$ is pure of weight 2 and Hodge type $(1,1)$.

Proof. $H^{2}(Y) \rightarrow H^{2}(\widetilde{Y})$ is injective.

Unfortunately, this direct approach we employed here is not as useful for computing $H_{3}(Y)$, since we need to understand the role of the codimension-two orbits. It is also not as useful for computing the map $H_{2}(\widetilde{Y}) \rightarrow H_{2}(Y)$ since $Y$ is singular. We find it convenient to organize the computation differently in order to address these issues.

\section{Integral homology of Calabi-Yau threefolds in toric varieties}

In this section we compute the maps $H_{*}(\widetilde{Y} ; \mathbb{Z}) \rightarrow H_{*}(Y ; \mathbb{Z})$ for $* \leq 3$. As we indicated at the end of the last section the direct approach inductively studying the intersection of $\widetilde{Y}$ with the orbits in $\mathbb{P}_{\mathcal{T}}$ of higher and higher codimension is not the best way to organize the argument. Rather one inductively considers the 
preimage of the intersection of $Y$ with the torus-orbits in $X$ of higher codimension. The reason is that the preimage of these intersection is a union of intersections of $\widetilde{Y}$ with orbits in $\mathbb{P}_{\mathcal{T}}$ of various dimension, but with the help of the combinatorial models for these preimages we are able to say a lot about their homology that is not apparent studying one orbit at a time in $\widetilde{Y}$.

3.1. The general set-up. Let $\Delta$ be a reflexive polytope of dimension $n$. Let $X=\mathbb{P}_{\Delta}$. Let $X_{i}$ denote the union of the $T_{N}$-orbits of dimension $\geq n-i$. We have the chain of inclusions

$$
X_{0} \subset X_{1} \subset X_{2} \subset X_{3} \subset X_{4} \subset \cdots \subset X_{n}=X .
$$

In particular $X_{0}$ is the open torus orbit and hence is isomorphic to $T_{N}$. The difference $X_{i} \backslash X_{i-1}$ is the union of orbits of complementary dimension, i.e.,

$$
X_{i} \backslash X_{i-1}=\coprod_{\operatorname{codim} \mathcal{O}=i} \mathcal{O}
$$

Denoting $\mathbb{P}_{\mathcal{F}}$, by $\widetilde{X}$ and by $\rho: \widetilde{X} \rightarrow X$ the induced resolution, we define $\widetilde{X}_{i}=$ $\rho^{-1}\left(X_{i}\right)$ so that we have

$$
\widetilde{X}_{0} \subset \widetilde{X}_{1} \subset \widetilde{X}_{2} \subset \widetilde{X}_{3} \subset \widetilde{X}_{4} \subset \cdots \subset \widetilde{X}_{n}=\tilde{X}
$$

As always $Y \subset X$ is either a generic section of $\mathcal{O}\left(D_{\infty}\right)$ if $\Delta$ has dimension 4 or $Y$ is a generic complete intersection arising from a NEF partition $V(\Delta)=$ $V_{1} \coprod \cdots \coprod V_{k}$ if $\Delta$ has dimension $3+k$. In the later case we assume each of the associated divisors $D_{i}$ are ample. In both cases we define the nested sequence of open subvarieties of $Y$

$$
Y_{0} \subset Y_{1} \subset Y_{2} \subset Y_{3}=Y
$$

where

$$
Y_{i}=Y \cap X_{i} .
$$

Let $Z_{i}$ be the intersection of $Y$ with all torus orbits of codimension $i$. Then

$$
Z_{i}=Y_{i} \backslash Y_{i-1}
$$

is a closed subvariety of $Y_{i}$. It is a disjoint union

$$
Z_{i}=\coprod_{\{f \mid \operatorname{dim}(f)=i-1\}} Z(f),
$$

where $Z(f)$ is $Y \cap \mathcal{O}_{f}$. We let $\nu_{i} \subset Y_{i}$ be a (closed) regular neighborhood of $Z_{i}$. We choose $\nu_{i}$ so that the projection $\pi: \nu_{i} \rightarrow Z_{i}$ is a proper, locally trivial fibration with compact fibers. The intersection of $\nu_{i}$ with any fiber is identified with a regular neighborhood of the cone point $0_{c}$ in $V(c)$. Then $\nu_{i}^{*}=\nu_{i} \cap Y_{i-1}$ is the complement of $0_{c}$ in this neighborhood, and $\partial \nu_{i}$ is a deformation retract of $\nu_{i}^{*}$. Of course, we have

We have

$$
Y_{i}=\nu_{i} \cup_{\nu_{i}^{*}} Y_{i-1}
$$

$$
\nu_{i}=\coprod_{\{f \mid \operatorname{dim}(f)=i-1\}} \nu(f)
$$

where $\nu(f)$ is the component of $\nu_{i}$ containing $\mathcal{O}_{f} \cap Y$. We see that the inclusion induces an identification

$$
H_{*}(\nu(f), \partial \nu(f))=H_{*}\left(\nu(f), \nu^{*}(f)\right) .
$$


Furthermore, each of these pairs is a locally trivial relative fibration over $Z(f)$ with fiber homotopy equivalent to the pair $\left(U\left(f, N_{f}\right), U^{*}\left(f, N_{f}\right)\right)$. The $T_{N}$-action produces a trivialization of this relative fiber bundle. The relative homology is then

$$
\begin{aligned}
H_{*}\left(\nu_{i}, \partial \nu_{i}\right) & \simeq \bigoplus_{\operatorname{dim} f=i a+b=*} \bigoplus_{a} H_{a}(Z(f)) \otimes H_{b}\left(V(f), V^{*}(f)\right) \\
& =\bigoplus_{\operatorname{dim} f=i a+b=*} \bigoplus_{a} H_{a}(Z(f)) \otimes H_{b-1}\left(V^{*}(f)\right) .
\end{aligned}
$$

We define $\tilde{Y}_{i}=\rho^{-1}\left(Y_{i}\right)$ for $i=1, \cdots, 3$, so that $\widetilde{Y}_{i}-\tilde{Y} \cap \widetilde{X}_{i}$. We obtain

$$
\widetilde{Y}_{0} \subset \widetilde{Y}_{1} \subset \widetilde{Y}_{2} \subset \widetilde{Y}_{3}=\widetilde{Y} .
$$

Let $\widetilde{\nu}_{i}=\rho^{-1}\left(\nu_{i}\right), \widetilde{\nu}_{i}^{*}=\rho^{-1}\left(\nu_{i}^{*}\right)$. We denote by $\widetilde{\nu}(f)$ and $\widetilde{\nu}^{*}(f)$ the preimages of $\nu(f)$ and $\nu^{*}(f)$ respectively. We also denote by $\rho_{i}: \widetilde{Y}_{i} \rightarrow Y_{i}$ the map induced by $\rho$. The pair $\left(\widetilde{\nu}(f), \widetilde{\nu}^{*}(f)\right) \rightarrow Z(f)$ is a relative fibration with fiber homotopy equivalent to the pair

$$
\left(\mathbb{P}_{\mathcal{F}_{f}^{\prime}\left(N_{f}\right)}, \mathbb{P}_{\mathcal{F}_{f}^{\prime}\left(N_{f}\right)} \backslash \mathcal{S}(f)\right)
$$

where $\mathcal{S}(f)$ is as given in Proposition 2.11] Again by Corollary 2.7 the $T_{N}$-action produces a trivialization of this bundle.

3.2. The comparison of $\widetilde{Y}_{1}$ and $Y_{1}$. We know that $\rho_{1}: \widetilde{Y}_{1} \rightarrow Y_{1}$ is an isomorphism.

3.3. The comparison of $\tilde{Y}_{2}$ and $Y_{2}$. To make this comparison, we need to understand the nature of $\widetilde{\nu}_{2}$ or equivalently, $\mathbb{P}_{\mathcal{F}_{e}^{\prime}\left(N_{e}\right)}$. For any edge $e$ of $\Delta$,

$$
\rho^{-1}\left(\mathcal{O}_{e}\right) \simeq \mathcal{O}_{e} \times \mathcal{S}(e) .
$$

Consider the one-cell complex dual to the restriction of $\mathcal{T}$ to $\stackrel{\circ}{e}$ : it has a one-cell for each vertex in $\stackrel{\circ}{e}$ and a zero-cell for each edge of $\left.\mathcal{T}\right|_{e}$. By Proposition [2.11 this dual cell complex is a combinatorial model for $\mathcal{S}(e)$ in the sense of Definition 2.10. Thus, $\mathcal{S}(e)$ is a chain of $\mathbb{P}^{1}$ 's, one irreducible component for each one-cell. Two irreducible components intersect if and only if the corresponding one-cells share a vertex, in which case the irreducible components meet in the single point which is the 0-dimensional orbit corresponding to this vertex. The smooth affine complex surface $\mathbb{P}_{\mathcal{F}_{e}^{\prime}\left(N_{e}\right)}$ deformation retracts onto $\mathcal{S}(e)$, and hence $H_{2}\left(\mathbb{P}_{\mathcal{F}_{e}^{\prime}\left(N_{e}\right)}\right)$ is identified with $\mathcal{A}_{\ell^{*}(e)}$, the root lattice of the Lie algebra $A_{\ell^{*}(e)}$. The intersection pairing on $\mathrm{H}_{2}$ of this surface agrees with the usual symmetric pairing on this root lattice given by the Cartan matrix. In particular, the pairing is non-degenerate and its adjoint has cyclic quotient of order $k(e)=\ell^{*}(e)+1$. This means that $H_{2}\left(\mathbb{P}_{\mathcal{F}_{e}^{\prime}\left(N_{e}\right)}, \partial \mathbb{P}_{\mathcal{F}_{e}^{\prime}\left(N_{e}\right)}\right)$ is identified with the dual lattice $\mathcal{A}_{\ell^{*}(e)}^{*}$ and the natural map

$$
H_{2}\left(\mathbb{P}_{\mathcal{F}_{e}^{\prime}\left(N_{e}\right)}\right) \rightarrow H_{2}\left(\mathbb{P}_{\mathcal{F}_{e}^{\prime}\left(N_{e}\right)}, \partial \mathbb{P}_{\mathcal{F}_{e}^{\prime}\left(N_{e}\right)}\right)
$$

is injective with cokernel, denoted $Q_{e}$, a cyclic group of order $k(e)$, see Example 2.5. Of course,

$$
\left(\widetilde{\nu}_{2}, \widetilde{\nu}_{2}^{*}\right) \simeq \coprod_{e \in E} Z(e) \times\left(\mathbb{P}_{\mathcal{F}_{e}^{\prime}\left(N_{e}\right)}, \mathbb{P}_{\mathcal{F}_{e}^{\prime}\left(N_{e}\right)} \backslash \mathcal{S}(e)\right)
$$


Define

$$
\begin{aligned}
\nu_{2}^{\prime} & =\overline{\nu_{2} \backslash\left(\nu_{2} \cap \nu_{3}\right)} & & \partial_{\mathrm{hor}} \nu_{2}^{\prime} & =\nu_{2} \cap \partial \nu_{3} \\
\widetilde{\nu}_{2}^{\prime} & =\rho^{-1}\left(\nu_{2}^{\prime}\right) & & \partial_{\mathrm{hor}} \widetilde{\nu}_{2}^{\prime} & =\rho^{-1}\left(\partial_{\mathrm{hor}} \nu_{2}^{\prime}\right) \\
Y_{2}^{\prime} & =\overline{Y_{2} \backslash\left(Y_{2} \cap \nu_{3}\right)} & , & Y_{1}^{\prime} & =\overline{Y_{1} \backslash\left(Y_{1} \cap\left(\nu_{2} \cup \nu_{3}\right)\right)} \\
\widetilde{Y}_{2}^{\prime} & =\rho^{-1}\left(Y_{2}^{\prime}\right) & , & \widetilde{Y}_{1}^{\prime} & =\rho^{-1}\left(Y_{1}^{\prime}\right) \\
Z^{\prime}(e) & =\frac{Z(e) \backslash\left(Z(e) \cap \nu_{3}\right)}{} & , & \partial Z^{\prime}(e) & =Z(e) \cap \partial \nu_{3}
\end{aligned}
$$

Note that $\partial Y_{2}^{\prime}=\partial \nu_{3}$. Also, $\widetilde{Y}_{2}^{\prime} \subset \widetilde{Y}_{2}$ is a homotopy equivalence, as are all the other similarly related primed and unprimed pairs. We denote by $\partial_{\mathrm{ver}} \nu_{2}^{\prime}$ the closure of $\partial \nu_{2}^{\prime} \backslash \partial_{\text {hor }} \nu_{2}^{\prime}$. Similarly, we define $\partial_{\text {ver }} \widetilde{\nu}_{2}^{\prime}$.

The pair $\left(\widetilde{Y}_{2}^{\prime}, \widetilde{Y}_{1}^{\prime}\right)$ is excisively equivalent to the pair $\left(\widetilde{\nu}_{2}^{\prime}, \partial \widetilde{\nu}_{2}^{\prime}\right)$. Analogously $\left(Y_{2}^{\prime}, Y_{1}^{\prime}\right)$ is excisively equivalent to $\left(\nu_{2}^{\prime}, \partial \nu_{2}^{\prime}\right)$. We have the long exact sequence of the pairs

$$
\begin{aligned}
& H_{*+1}\left(\widetilde{\nu}_{2}^{\prime}, \partial_{\text {ver }} \widetilde{\nu}_{2}^{\prime}\right) \rightarrow H_{*}\left(\widetilde{Y}_{1}^{\prime}\right) \rightarrow H_{*}\left(\widetilde{Y}_{2}^{\prime}\right) \rightarrow H_{*}\left(\widetilde{\nu}_{2}^{\prime}, \partial_{\text {ver }} \widetilde{\nu}_{2}^{\prime}\right) \rightarrow H_{*-1}\left(\widetilde{Y}_{1}^{\prime}\right) \\
& \underset{H_{*+1}\left(\nu_{2}^{\prime}, \partial_{\mathrm{ver}} \nu_{2}^{\prime}\right)}{\downarrow} \rightarrow \underset{H_{*}\left(Y_{1}^{\prime}\right)}{\|} \rightarrow \underset{H_{*}}{\stackrel{\downarrow}{\downarrow}\left(Y_{2}^{\prime}\right)} \rightarrow H_{*}\left(\nu_{2}^{\prime}, \partial_{\mathrm{ver}} \nu_{2}^{\prime}\right) \rightarrow H_{*-1}\left(Y_{1}^{\prime}\right) \\
& \downarrow \\
& 0
\end{aligned}
$$

Our descriptions of $\widetilde{\nu}_{2}$ and $\nu_{2}$ lead to the following:

Clatm 3.1.

$$
\begin{aligned}
& H_{*}\left(\widetilde{\nu}_{2}^{\prime}, \partial_{\mathrm{ver}} \widetilde{\nu}_{2}^{\prime}\right)=\bigoplus_{e}\left(H_{*-2}\left(Z^{\prime}(e)\right) \otimes \mathcal{A}_{\ell^{*}(e)}^{*}\right) \oplus \bigoplus_{e}\left(H_{*-4}\left(Z^{\prime}(e)\right) \otimes \mathbb{Z}\right) \\
& H_{*}\left(\nu_{2}^{\prime}, \partial_{\mathrm{ver}} \nu_{2}^{\prime}\right)=\bigoplus_{e}\left(H_{*-2}\left(Z^{\prime}(e)\right) \otimes Q_{e}\right) \oplus \bigoplus_{e}\left(H_{*-4}\left(Z^{\prime}(e)\right) \otimes \mathbb{Z}\right)
\end{aligned}
$$

and the projection map

$$
\rho_{*}: H_{*}\left(\widetilde{\nu}_{2}^{\prime}, \partial_{\mathrm{ver}} \widetilde{\nu}_{2}^{\prime}\right) \rightarrow H_{*}\left(\nu_{2}^{\prime}, \partial_{\mathrm{ver}} \nu_{2}^{\prime}\right)
$$

is the natural one.

Thus, $\rho_{*}$ is surjective with kernel $\bigoplus_{e} H_{*-2}\left(Z^{\prime}(e)\right) \otimes \mathcal{A}_{\ell^{*}(e)}$.

An elementary diagram chase in the long exact sequence above shows that $H_{2}\left(\widetilde{Y}_{2}^{\prime}\right) \rightarrow H_{2}\left(Y_{2}^{\prime}\right)$ is surjective and its kernel is identified with the kernel of

$$
H_{2}\left(\widetilde{\nu}_{2}^{\prime}, \partial_{\text {ver }} \widetilde{\nu}_{2}^{\prime}\right) \rightarrow H_{2}\left(\nu_{2}^{\prime}, \partial_{\text {ver }} \nu_{2}^{\prime}\right) .
$$

Thus, we have established the following relationship between $H_{*}\left(\widetilde{Y}_{2}\right)$ and $H_{*}\left(Y_{2}\right)$ :

Lemma 3.2. There is an exact sequence

$$
0 \rightarrow \bigoplus_{e} H_{*-2}(Z(e)) \otimes \mathcal{A}_{\ell^{*}(e)} \rightarrow H_{*}\left(\tilde{Y}_{2}\right) \rightarrow H_{*}\left(Y_{2}\right) \rightarrow 0 .
$$

The kernel is represented by the fundamental classes of the $\mathbb{P}(\ell)$-bundles over cycle representatives for the classes in $H_{*-2}(Z(e))$.

Exactly the same argument can be applied to $\partial \widetilde{\nu}_{3} \rightarrow \partial \nu_{3}$. Recall from the definition that $\partial Z^{\prime}(e)$ is contained in $\partial \nu_{3}$. For any edge $e$ and any two-face $f$ containing $e$ in its closure denote by $\partial_{f} Z^{\prime}(e)$ the components of $\partial Z(e)$ given by $\partial \nu(f) \cap Z^{\prime}(e)$, so that $\partial Z^{\prime}(e)=\coprod_{f \mid e \prec f} \partial_{f} Z^{\prime}(e)$. 
LEMMA 3.3. For any two-face $f$ of $\partial \Delta$ there is a short exact sequence

$$
0 \rightarrow \bigoplus_{e \in E ; e \prec f} H_{*-2}\left(\partial_{f} Z^{\prime}(e)\right) \otimes \mathcal{A}_{\ell^{*}(e)} \rightarrow H_{*}(\partial \widetilde{\nu}(f)) \rightarrow H_{*}(\partial \nu(f)) \rightarrow 0 .
$$

The kernel is represented by the fundamental classes of the $\mathbb{P}^{1}(\ell)$-bundles over the cycles in $\partial_{f} Z^{\prime}(e)$ for the classes in $H_{*-2}\left(\partial_{f} Z^{\prime}(e)\right)$.

In order to carry out the next step in the comparison, we need to understand the relative version, that is to say the map $H_{*}\left(\widetilde{Y}_{2}^{\prime}, \partial \widetilde{\nu}_{3}\right) \rightarrow H_{*}\left(Y_{2}^{\prime}, \partial \nu_{3}\right)$.

Proposition 3.4. $H_{*}\left(\widetilde{Y}_{2}^{\prime}, \partial \widetilde{\nu}_{3}\right) \rightarrow H_{*}\left(Y_{2}^{\prime}, \partial \nu_{3}\right)$ is an isomorphism for $* \leq 2$ and is surjective with kernel $\bigoplus_{e \in E} H_{1}\left(Z^{\prime}(e), \partial Z^{\prime}(e)\right) \otimes \mathcal{A}_{\ell^{*}(e)}$ for $*=3$.

Proof. We consider $\partial \widetilde{\nu}_{3} \subset\left(\partial \widetilde{\nu}_{3} \cup \widetilde{\nu}_{2}^{\prime}\right) \subset \widetilde{Y}_{2}^{\prime}$ and $\partial \nu_{3} \subset\left(\partial \nu_{3} \cup \nu_{2}^{\prime}\right) \subset Y_{2}^{\prime}$. Of course, $\left(\widetilde{Y}_{2}^{\prime}, \partial \widetilde{\nu}_{3} \cup \widetilde{\nu}_{2}^{\prime}\right) \simeq\left(\widetilde{Y}_{1}^{\prime}, \partial \widetilde{Y}_{1}^{\prime}\right)$ and $\left(Y_{2}^{\prime}, \partial \nu_{3} \cup \nu_{2}^{\prime}\right) \simeq\left(Y_{1}^{\prime}, \partial Y_{1}^{\prime}\right)$. Thus we have

$$
\begin{array}{ccccccccc}
H_{*+1}\left(\widetilde{Y}_{1}^{\prime}, \partial \widetilde{Y}_{1}^{\prime}\right) & \rightarrow & H_{*}\left(\widetilde{\nu}_{2}^{\prime}, \partial_{\text {hor }} \widetilde{\nu}_{2}^{\prime}\right) & \rightarrow & H_{*}\left(\widetilde{Y}_{2}^{\prime}, \partial \widetilde{\nu}_{3}\right) & \rightarrow & H_{*}\left(\widetilde{Y}_{1}^{\prime}, \partial \widetilde{Y}_{1}^{\prime}\right) & \rightarrow & H_{*-1}\left(\widetilde{\nu}_{2}^{\prime}, \partial_{\text {hor }} \widetilde{\nu}_{2}^{\prime}\right) \\
H_{*+1}\left(Y_{1}^{\prime}, \partial Y_{1}^{\prime}\right) & \rightarrow & H_{*}\left(\nu_{2}^{\prime}, \partial_{\text {hor }} \nu_{2}^{\prime}\right) & \rightarrow & H_{*}\left(Y_{2}^{\prime}, \partial \nu_{3}\right) & \rightarrow & H_{*}\left(Y_{1}^{\prime}, \partial Y_{1}^{\prime}\right) & \rightarrow & H_{*-1}\left(\nu_{2}^{\prime}, \partial_{\mathrm{hor}} \nu_{2}^{\prime}\right) .
\end{array}
$$

Again, the description of $\widetilde{\nu}_{2}$ and $\nu_{2}$ leads immediately to the following:

Claim 3.5.

$$
\begin{aligned}
& H_{*}\left(\widetilde{\nu}_{2}^{\prime}, \partial_{\mathrm{hor}} \widetilde{\nu}_{2}^{\prime}\right)=\bigoplus_{e \in E}\left(H_{*-2}\left(Z^{\prime}(e), \partial Z^{\prime}(e)\right) \otimes \mathcal{A}_{\ell^{*}(e)}\right) \oplus \bigoplus_{e \in E} H_{*}\left(Z^{\prime}(e), \partial Z^{\prime}(e)\right) \\
& H_{*}\left(\nu_{2}^{\prime}, \partial_{\mathrm{hor}} \nu_{2}^{\prime}\right)=\bigoplus_{e \in E} H_{*}\left(Z^{\prime}(e), \partial Z^{\prime}(e)\right)
\end{aligned}
$$

and the projection map induces the natural one on homology.

Thus, $H_{*}\left(\widetilde{\nu}_{2}^{\prime}, \partial_{\text {hor }} \widetilde{\nu}_{2}^{\prime}\right) \rightarrow H_{*}\left(\nu_{2}^{\prime}, \partial_{\text {hor }} \nu_{2}^{\prime}\right)$ is surjective with kernel $H_{*-2}\left(Z^{\prime}(e), \partial Z^{\prime}(e)\right)$. In particular, $H_{*}\left(\widetilde{\nu}_{2}^{\prime}, \partial_{\text {hor }} \widetilde{\nu}_{2}^{\prime}\right) \rightarrow H_{*}\left(\nu_{2}^{\prime}, \partial_{\text {hor }} \nu_{2}^{\prime}\right)$ is an isomorphism for $* \leq 2$. Plugging this into the commutative diagram, the five lemma tells us that $H_{*}\left(\widetilde{Y}_{2}^{\prime}, \partial \widetilde{\nu}_{3}\right) \rightarrow$ $H_{*}\left(Y_{2}^{\prime}, \partial \nu_{3}\right)$ is an isomorphism for $* \leq 2$.

Now we consider $*=3$.

$$
\begin{aligned}
& H_{4}\left(\widetilde{Y}_{1}^{\prime}, \partial \widetilde{Y}_{1}^{\prime}\right) \rightarrow H_{3}\left(\widetilde{\nu}_{2}^{\prime}, \partial_{\mathrm{hor}} \widetilde{\nu}_{2}^{\prime}\right) \rightarrow H_{3}\left(\widetilde{Y}_{2}^{\prime}, \partial \widetilde{\nu}_{3}\right) \rightarrow H_{3}\left(\widetilde{Y}_{1}^{\prime}, \partial \widetilde{Y}_{1}^{\prime}\right) \rightarrow H_{2}\left(\widetilde{\nu}_{2}^{\prime}, \partial_{\mathrm{hor}} \widetilde{\nu}_{2}^{\prime}\right) \\
& \downarrow=1 \quad \downarrow \quad \downarrow \quad \downarrow=\quad \downarrow \simeq \\
& H_{4}\left(Y_{1}^{\prime}, \partial Y_{1}^{\prime}\right) \rightarrow \quad 0 \quad \rightarrow \quad H_{3}\left(Y_{2}^{\prime}, \partial \nu_{3}\right) \rightarrow H_{3}\left(Y_{1}^{\prime}, \partial Y_{1}^{\prime}\right) \rightarrow H_{2}\left(\nu_{2}^{\prime}, \partial_{\mathrm{hor}} \nu_{2}^{\prime}\right) \text {. }
\end{aligned}
$$

A standard diagram chase shows that $H_{3}\left(\widetilde{Y}_{2}^{\prime}, \partial \widetilde{\nu}_{3}\right) \rightarrow H_{3}\left(Y_{2}^{\prime}, \partial \nu_{3}\right)$ is onto, with kernel the image of $H_{3}\left(\widetilde{\nu}_{2}^{\prime}, \partial_{\text {hor }} \widetilde{\nu}_{2}^{\prime}\right) \rightarrow H_{3}\left(\widetilde{Y}_{2}^{\prime}, \partial \widetilde{\nu}_{3}\right)$. Of course,

$$
H_{3}\left(\widetilde{\nu}_{2}^{\prime}, \partial_{\mathrm{hor}} \widetilde{\nu}_{2}^{\prime}\right)=\bigoplus_{e \in E} H_{1}\left(Z^{\prime}(e), \partial Z^{\prime}(e)\right) \otimes \mathcal{A}_{\ell^{*}(e)}
$$

To complete the argument, we need to know that $H_{4}\left(\widetilde{Y}_{1}^{\prime}, \partial \widetilde{Y}_{1}^{\prime}\right) \rightarrow H_{3}\left(\widetilde{\nu}_{2}^{\prime}, \partial_{\text {hor }} \widetilde{\nu}_{2}^{\prime}\right)$ is the trivial map. That is the content of the next lemma.

LEMMA 3.6.

$$
H_{4}\left(\widetilde{Y}_{1}^{\prime}, \partial \widetilde{Y}_{1}^{\prime}\right) \rightarrow H_{3}\left(\widetilde{\nu}_{2}^{\prime}, \partial_{\text {hor }} \widetilde{\nu}_{2}^{\prime}\right)
$$

is the trivial map. 
Proof. The morphism factors as

$H_{4}\left(\widetilde{Y}_{1}^{\prime}, \partial \widetilde{Y}_{1}^{\prime}\right) \rightarrow H_{3}\left(\partial \widetilde{Y}_{1}^{\prime}\right) \rightarrow H_{3}\left(\partial \widetilde{Y}_{1}^{\prime}, \widetilde{\nu}_{3} \cap \partial \widetilde{Y}_{1}^{\prime}\right) \simeq H_{3}\left(\partial_{\text {ver }} \widetilde{\nu}_{2}^{\prime}, \partial_{\text {hor }} \partial_{\text {ver }} \widetilde{\nu}_{2}^{\prime}\right) \rightarrow H_{3}\left(\widetilde{\nu}_{2}^{\prime}, \partial_{\text {hor }} \widetilde{\nu}_{2}^{\prime}\right)$,

where we introduce the notation

$$
\begin{aligned}
\partial_{\text {ver }} \nu_{2}^{\prime} & =\nu_{2}^{\prime} \cap Y_{1}^{\prime} \\
\partial_{\text {hor }} \partial_{\text {ver }} \nu_{2}^{\prime} & =\partial_{\text {ver }} \partial_{\text {hor }} \nu_{2}^{\prime}=\partial\left(\nu_{2}^{\prime} \cap \partial \nu_{3}\right)=\partial_{\text {ver }} \nu_{2}^{\prime} \cap \partial \nu_{3} .
\end{aligned}
$$

The isomorphism is from excision. The pair $\left(\widetilde{\nu}_{2}^{\prime}, \partial_{\text {hor }} \widetilde{\nu}_{2}^{\prime}\right)$ is a locally trivial fiber bundle over $\coprod_{e}\left(Z^{\prime}(e), \partial Z^{\prime}(e)\right)$ with fiber over any $x \in Z^{\prime}(e)$ a regular neighborhood of $\mathcal{A}_{\ell^{*}(e)}$. Hence, $\left.H_{3}\left(\widetilde{\nu}_{2}^{\prime}, \partial_{\mathrm{hor}} \widetilde{\nu}_{2}^{\prime}\right)\right) \simeq \bigoplus_{e} H_{1}\left(Z^{\prime}(e), \partial Z^{\prime}(e)\right) \otimes \mathcal{A}_{\ell^{*}(e)}$ is free abelian. On the other hand, $\left(\partial_{\text {ver }} \widetilde{\nu}_{2}^{\prime}, \partial_{\text {hor }} \partial_{\text {ver }} \widetilde{\nu}_{2}^{\prime}\right)$ is a locally trivial fiber bundle over $\coprod_{e}\left(Z^{\prime}(e), \partial Z^{\prime}(e)\right)$ with fiber over any $x \in Z^{\prime}(e)$ identified with the boundary of a regular neighborhood of the cone point $0_{e}$ in $V(e)$. The latter is the lens space $L(n(e),-1)$. Thus, $H_{3}\left(\partial_{\text {ver }} \widetilde{\nu}_{2}^{\prime}, \partial_{\text {hor }} \partial_{\text {ver }} \widetilde{\nu}_{2}^{\prime}\right)=\bigoplus_{e} H_{2}\left(Z^{\prime}(e), \partial Z^{\prime}(e)\right) \otimes \operatorname{Tor}_{\ell^{*}(e)}$ is a torsion group. Hence, the morphism in Equation (3.1) is the zero map.

This completes the proof of Proposition 3.4

Note that the three inclusions of

$$
\begin{aligned}
\left(\widetilde{Y}_{2}^{\prime}, \partial \widetilde{\nu}_{3}\right) & \hookrightarrow\left(\widetilde{Y}, \widetilde{\nu}_{3}\right) \\
\left(Y_{2}^{\prime}, \partial \nu_{3}\right) & \hookrightarrow\left(Y_{2}, \nu_{3}\right) \\
\left(Z^{\prime}(e), \partial Z^{\prime}(e)\right) & \hookrightarrow\left(Z(e), Z(e) \cap \nu_{3}\right)
\end{aligned}
$$

each consist of excisive pairs.

3.4. The comparison of $\widetilde{Y}$ and $Y$. In the previous subsection, to study the relationship between $\widetilde{Y}_{2}$ and $Y_{2}$, for each edge $e$ of $\partial \Delta$ we described $\mathcal{S}(e)$ in terms of its combinatorial model which is the cell complex dual to $\left.\mathcal{T}\right|_{e}$. Analogously, in order to understand the relationship between $\widetilde{Y}$ and $Y$ we need to understand the homology of the surfaces $\mathcal{S}(f)$ for two-faces $f$ of $\partial \Delta$. Of course by Proposition 2.11 we have analogous result for each two-face $f$ : the combinatorial model for $\mathcal{S}(f)$ is the cell complex dual to the triangulation of $\stackrel{\circ}{f}$. We study $\mathcal{S}(f)$ using this cell complex.

Let $\Sigma$ be a finite, connected cell complex dual to the triangulation of the interior of a two-face. Then every cell of $\Sigma$ has dimension $\leq 2$, every vertex $v$ has valence, denoted $o(v)$, which is $\leq 3$, and every edge has two vertices. Let $\mathcal{S}$ be a compact algebraic variety of dimension $\leq 2$ such that $\Sigma$ is a combinatorial model for $\mathcal{S}$. It follows from the definition that for every edge $\alpha$ of $\Sigma$ the algebraic subset $\bar{S}_{\alpha}$ of $\mathcal{S}$ is isomorphic to $\mathbb{P}^{1}$. We also denote this subset by $\mathbb{P}^{1}(\alpha)$.

Define $\Sigma_{2} \subset \Sigma$ to be the sub-cell complex which is the closure of the union of the two-cells of $\Sigma$. Let $\Sigma_{1} \subset \Sigma$ be the sub-cell complex consisting the closure of the union of all edges of $\Sigma$ that are not contained in $\Sigma_{2}$.

Notice that $\Sigma_{2}$ is a disjoint union of contractible homogeneously two-dimensional complexes, and $\Sigma_{1}$ is a disjoint union of trees. Furthermore, $\Sigma_{2} \cap \Sigma_{1}$ consists of a set of trivalent vertices of $\Sigma$. If $A$ is a component of $\Sigma_{2}$ we denote by $\mathcal{S}_{A}$ the corresponding, possibly reducible, surface in $\mathcal{S}$. If $X$ is a component of $\Sigma_{1}$ we denote by $\mathcal{S}_{X}$ the corresponding curve (which is a union of irreducible rational components) in $\mathcal{S}$. We denote by $\mathcal{C}_{2}$ the set of two-cells in $\Sigma$, by $\mathcal{E}_{2}$ the set of edges in $\Sigma_{2}$, and 

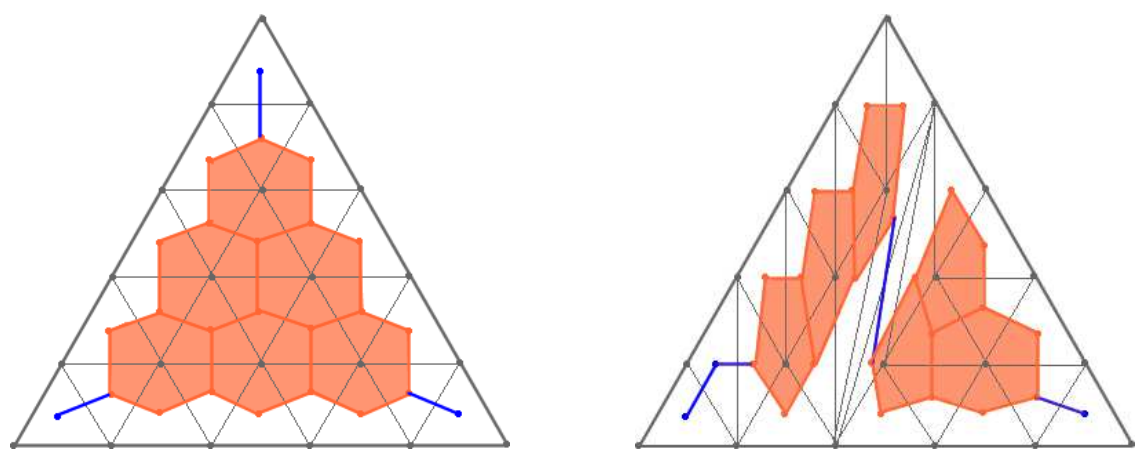

FIGURE $3 . \Sigma_{1}$ and $\Sigma_{2}$ for the triangulations of Figure 2

by $\mathcal{B}_{2}$ the set of interior edges of $\Sigma_{2}$. Lastly, we denote by $\mathcal{E}_{1}$ the set of edges in $\Sigma_{1}$.

The following is immediate from the Meyer-Vietoris sequence.

Lemma 3.7. Let $\Sigma$ be a combinatorial model for $\mathcal{S}$ as above. Then

$$
\begin{aligned}
& H_{0}(\mathcal{S})=\mathbb{Z} \\
& H_{1}(\mathcal{S})=\bigoplus_{A \in \pi_{0}\left(\Sigma_{2}\right)} H_{1}\left(\mathcal{S}_{A}\right) \\
& H_{2}(\mathcal{S})=\bigoplus_{A \in \pi_{0}\left(\Sigma_{2}\right)} H_{2}\left(\mathcal{S}_{A}\right) \oplus \bigoplus_{\alpha \in \mathcal{E}_{1}} H_{2}\left(\mathbb{P}^{1}(\alpha)\right) \\
& H_{3}(\mathcal{S})=\bigoplus_{A \in \pi_{0}\left(\Sigma_{2}\right)} H_{3}\left(\mathcal{S}_{A}\right) \\
& H_{4}(\mathcal{S})=\bigoplus_{A \in \pi_{0}\left(\Sigma_{2}\right)} H_{4}\left(\mathcal{S}_{A}\right) .
\end{aligned}
$$

Recall that

$$
\mathcal{S}(f)=\rho^{-1}\left(0_{f}\right) .
$$

We now wish to compute the cohomology of $\left(\mathbb{P}_{\mathcal{F}_{f}^{\prime}\left(N_{f}\right)}, \mathbb{P}_{\mathcal{F}_{f}^{\prime}\left(N_{f}\right)} \backslash \mathcal{S}(f)\right)$. Of course, by Proposition 2.11 $\Sigma\left(\mathcal{F}_{f}^{\prime}\right)$ is a combinatorial model for $\mathcal{S}(f)$. Clearly, $\Sigma\left(\mathcal{F}_{f}^{\prime}\right)$ is identified with a polyhedral subset of the face $f$ of $\Delta$ and hence is embedded in the plane spanned by $f$. To simplify notation we set $\Sigma=\Sigma\left(\mathcal{F}_{f}^{\prime}\right)$ and $S=\mathcal{S}(f)$.

Consider the pair

$$
\left(\mathbb{P}_{\mathcal{F}_{f}^{\prime}\left(N_{f}\right)}, \mathbb{P}_{\mathcal{F}_{f}^{\prime}\left(N_{f}\right)} \backslash S\right) .
$$

Since $\mathbb{P}_{\mathcal{F}_{f}^{\prime}\left(N_{f}\right)}$ deformation retracts to $S$, by Lefschetz duality, we have

$$
H^{i}\left(\mathbb{P}_{\mathcal{F}_{f}^{\prime}\left(N_{f}\right)}, \mathbb{P}_{\mathcal{F}_{f}^{\prime}\left(N_{f}\right)} \backslash S\right)=H_{6-i}(S) .
$$

The following lemma computes these groups:

Lemma 3.8. For any $f \in F$, we have

$$
\begin{aligned}
& H_{6}(S)=0 \\
& H_{4}(S)=\bigoplus_{\left\{c \in \mathcal{C}_{2}\right\}} \mathbb{Z}\left[\bar{S}_{c}\right] \\
& H_{0}(S)=\mathbb{Z},
\end{aligned}
$$

and all odd homology groups vanish. Furthermore, $\mathrm{H}_{2}(S)$ is free abelian with rank

$$
b_{2}(S)=\ell^{*}(f)+\sum_{\{\text {edges } e \mid e \prec f\}} \ell^{*}(e)+v_{f}-3 .
$$


Proof. Let $\mathcal{T}_{f}$ be the induced triangulation of $f$. We say that an edge of a two-dimensional triangulation of a surface is free if it is the face of exactly one two-simplex. There are three types of simplifications we wish to perform on triangulations of surfaces:

Type A Remove a two-simplex whose closure meets the boundary in exactly one edge and remove that free edge as well.

Type B Remove a two-simplex with exactly two free edges and remove both free edges and the vertex they have in common.

Type C Remove a two-simplex with three free edges, all its edges, and all its vertices.

A triangulated surface with boundary is said to be shellable if there is a finite sequence of these simplifications that reduces it to the empty triangulation. Notice that each of these operations replaces a triangulated surface by a triangulated subsurface with one fewer two-simplex. As we perform a sequence of these operations we obtain a decreasing sequence of triangulations on subsurfaces of $f$. Dual to this sequence of triangulations is the sequence of dual cell complexes. Each time we remove simplices from one of the triangulations, we remove their dual cells from the dual cell complex. Thus, we produce a decreasing sequence of subcell complexes $\Sigma=\Gamma_{0} \supset \Gamma_{1} \supset \cdots \supset \Gamma_{m}=\emptyset$. Corresponding to the sequence of subcell complexes there is a decreasing sequence of $T_{N_{f}}$-invariant locally closed algebraic subsets $S=\mathcal{S}_{0} \supset \mathcal{S}_{1} \supset \cdots \supset \mathcal{S}_{m}=\emptyset$ : As we remove a cell of $\Gamma_{i}$ we remove the corresponding $T_{N_{f}}$ orbit from $\mathcal{S}_{i}$. Suppose that $\mathcal{S}_{i+1}$ is obtained from $\mathcal{S}_{i}$ by an operation of Type A. Then $\mathcal{S}_{i+1}$ is obtained by removing a contractible subset of a closed irreducible (complex) surface component of $\mathcal{S}_{i}$. The subset is disjoint from all other irreducible components of $\mathcal{S}_{i}$. Hence, $b_{4}\left(\mathcal{S}_{i+1}\right)=b_{4}\left(\mathcal{S}_{i}\right)-1$, and all other homology groups are the same. Suppose instead that $\mathcal{S}_{i+1}$ is obtained from $\mathcal{S}_{i}$ by an operation of Type B. Then $\mathcal{S}_{i+1}$ is obtained by removing a contractible subset of an irreducible component of $\mathcal{S}_{i}$ isomorphic to $\mathbb{P}^{1}$. Again, the subset is disjoint from all other irreducible components of $\mathcal{S}_{i}$. Hence, $b_{2}\left(\mathcal{S}_{i+1}\right)=b_{2}\left(\mathcal{S}_{i}\right)-1$, and all other homology groups are the same. Lastly, suppose that $\mathcal{S}_{i+1}$ is obtained from $\mathcal{S}_{i}$ by an operation of Type C. Then $\mathcal{S}_{i+1}$ is obtained by removing an isolated point of $\mathcal{S}_{i}$. Hence, $b_{0}\left(\mathcal{S}_{i+1}\right)=b_{0}\left(\mathcal{S}_{i}\right)-1$, and all other homology groups are the same.

Claim 3.9. Let $\tau$ be a compact triangulated contractible surface. Then there is a sequence of operations of Types $A, B$, and $C$ that reduce $\tau$ to the empty surface.

Proof. The argument is by induction on the number of two-simplices in $\tau$. If there is a two-simplex of $\tau$ whose intersection with the boundary is an edge, then we can perform an operation of Type A on this two-simplex producing a contractible surface with one fewer two-simplex. Suppose there are no such two-simplices. Then every two-simplex that contains an edge of the boundary has all its vertices in the boundary. Orient the boundary and let $\sigma$ be a two-simplex containing an edge $\alpha$ of the boundary with third vertex $p_{\alpha}$. Define the distance from $\alpha$ to $p_{\alpha}$ along the boundary to be the number of edges moving in the positive direction that separate $\alpha$ from $p_{\alpha}$. If this distance is 1 , then $\sigma$ has at least two edges in the boundary. If it has exactly two, then we can perform an operation of Type $\mathrm{B}$ on $\sigma$. If it has all three edges in the boundary, then we can perform an operation of Type $\mathrm{C}$ on $\sigma$. If the distance along the boundary from $\alpha$ to $p_{\alpha}$ is greater than one, let $\alpha^{\prime}$ be the edge next to $\alpha$ in positive direction along the boundary. Clearly, the distance along 
the boundary from $\alpha^{\prime}$ to $p_{\alpha^{\prime}}$ is less than that from $\alpha$ to $p_{\alpha}$. Eventually we arrive at an edge $\alpha_{0}$ with the distance from $\alpha_{0}$ to $p_{\alpha_{0}}$ being 1 , completing the induction.

Given this, it follows from the above description and induction that all the homology groups of $S$ are free abelian and all those except $H_{4}(S), H_{2}(S)$, and $H_{0}(S)$ vanish. The groups $H_{4}$ and $H_{0}$ are clearly as stated. The rank of $H_{2}$ is the number of operations of Type B. A direct combinatorial argument shows that the number of operations of Type B is as given in the statement.

Since $\left(\widetilde{Y}, \widetilde{Y}_{2}^{\prime}\right)$ is excisively equivalent to $\left(\widetilde{\nu}_{3}, \partial \widetilde{\nu}_{3}\right)$, we have:

Corollary 3.10.

$$
H_{i}\left(\widetilde{\nu}_{3}, \partial \widetilde{\nu}_{3}\right) \simeq H_{i}\left(\widetilde{Y}, \widetilde{Y}_{2}^{\prime}\right)=\bigoplus_{f \in F} H_{0}\left(Y \cap \mathcal{O}_{f}\right) \otimes H_{i}\left(\mathbb{P}_{\mathcal{F}_{f}^{\prime}\left(N_{f}\right)}, \mathbb{P}_{\mathcal{F}_{f}^{\prime}\left(N_{f}\right)} \backslash \mathcal{S}(f)\right) .
$$

Of course, since $\widetilde{\nu}_{3}$ is homotopy equivalent to $\mathcal{S}(f)$, we see that $H_{\text {odd }}\left(\widetilde{\nu}_{3}\right)=0$ and $H_{e v}\left(\widetilde{\nu}_{3}\right)$ is free abelian.

\subsection{The computation of $\rho_{*}: H_{1}(\widetilde{Y}) \rightarrow H_{1}(Y)$.}

Proposition 3.11. The morphism

$$
\rho_{*}: H_{1}(\widetilde{Y}) \rightarrow H_{1}(Y)
$$

is an isomorphism, and $H_{1}(\widetilde{Y})$ is identified with the quotient of $N$ by the lattice spanned by the 2-skeleton of $\partial \Delta$.

Proof. By Lemma 3.2 the restriction of $\rho$ induces an isomorphism

$$
H_{1}\left(\widetilde{Y}_{2}\right) \stackrel{\cong}{\longrightarrow} H_{1}\left(Y_{2}\right) \text {. }
$$

We have the exact sequence

$$
\begin{aligned}
& \cdots \quad \rightarrow \quad H_{2}(\widetilde{Y}) \rightarrow H_{2}\left(\widetilde{Y}, \widetilde{Y}_{2}\right) \rightarrow H_{1}\left(\widetilde{Y}_{2}\right) \rightarrow H_{1}(\widetilde{Y}) \rightarrow \quad \rightarrow \quad 0
\end{aligned}
$$

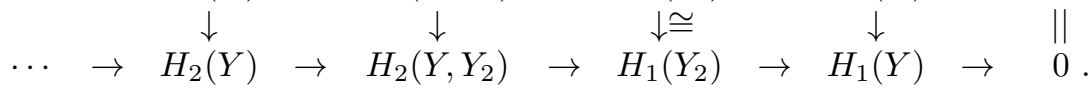

To prove that the map $\rho_{*}: H_{1}(\widetilde{Y}) \rightarrow H_{1}(Y)$ is an isomorphism we need only see that the natural map $H_{2}\left(\widetilde{Y}, \widetilde{Y}_{2}\right) \rightarrow H_{2}\left(Y, Y_{2}\right)$ is onto. By excision, it is equivalent to show that $H_{2}\left(\widetilde{\nu}_{3}, \partial \widetilde{\nu}_{3}\right) \rightarrow H_{2}\left(\nu_{3}, \partial \nu_{3}\right)$ is onto.

Claim 3.12. $H_{2}\left(\widetilde{\nu}_{3}, \partial \widetilde{\nu}_{3}\right) \rightarrow H_{2}\left(\nu_{3}, \partial \nu_{3}\right)$ is onto and the range is torsion.

Proof. Since $\nu_{3}$ is a disjoint union of cones, $H_{2}\left(\nu_{3}, \partial \nu_{3}\right) \cong H_{1}\left(\partial \nu_{3}\right)$.

Lemma 3.3 shows that $H_{1}\left(\partial \widetilde{\nu}_{3}\right) \rightarrow H_{1}\left(\partial \nu_{3}\right)$ is onto. Thus, to complete the proof of surjectivity, we need only see that $H_{2}\left(\widetilde{\nu}_{3}, \partial \widetilde{\nu}_{3}\right) \rightarrow H_{1}\left(\partial \widetilde{\nu}_{3}\right)$ is surjective, but that follows because $H_{1}\left(\widetilde{\nu}_{3}\right)=0$ by Corollary 3.10

To establish that $H_{2}\left(\nu_{3}, \partial \nu_{3}\right)$ is torsion, we must show that for each two-face $f$, $H_{1}\left(\partial \nu_{3}(f)\right)=0$. Of course, $\partial \nu_{3}(f)$ is homotopy-equivalent to $V(f)^{*}=V(f) \backslash 0_{f}$. But $V(f)$ is a three-dimensional toric variety and $V(f)^{*}$ is the union of all the torus orbits of codimension $<3$, i.e., $V(f)^{*}=V(f)_{2}$ (with notation analogous to that for the $\left.X_{i} \subset X\right)$. The result now follows from the observations that $V(f)_{0}=T_{N_{f}}$, that

$$
H_{1}\left(V(f)_{1}\right)=\frac{N_{f}}{\operatorname{Span} V(f)}
$$


(which is torsion) by the exact sequence for the pair $V(f)_{0} \hookrightarrow V(f)_{1}$, and that

$$
H_{1}\left(V(f)_{2}\right)=\frac{N_{f}}{\left\langle L \cap f^{(1)}\right\rangle}
$$

by the exact sequence for the pair $V(f)_{1} \hookrightarrow V(f)_{2}$.

This completes the proof of Proposition 3.11

3.6. The computation of $\rho_{*}: H_{2}(\widetilde{Y}) \rightarrow H_{2}(Y)$. In Corollary 2.22 we showed that in the hypersurface case

$$
\operatorname{rank} H_{2}(\tilde{Y})=\# V+\sum_{e} \ell^{*}(e)+\sum_{f} \ell^{*}(f)\left(1+\ell^{*}\left(f^{\vee}\right)\right)-4
$$

and in the complete intersection case that

$$
\operatorname{rank} H_{2}(\widetilde{Y})=\# V+\sum_{e} \ell^{*}(e)+\sum_{f} \ell^{*}(f)\left(1+\sum_{J \subset I ; J \neq \emptyset}(-1)^{3-|J|} \ell^{*}\left(\sum_{j \in J} f_{j}^{\vee}\right)-n .\right.
$$

We also gave a formula in terms of the lattice and $\Delta$ for the torsion part of this group. The new result here is:

Proposition 3.13. The morphism

$$
\rho_{*}: H_{2}(\widetilde{Y}) \rightarrow H_{2}(Y)
$$

is onto.

Proof. We begin by computing with the two excisive triples

$$
\begin{aligned}
\widetilde{Y}_{2}^{\prime} \subset \widetilde{Y} \rightarrow\left(\widetilde{Y}, \widetilde{Y}_{2}^{\prime}\right) & \simeq\left(\widetilde{\nu}_{3}, \partial \widetilde{\nu}_{3}\right) \\
Y_{2}^{\prime} \subset Y \rightarrow\left(Y, Y_{2}^{\prime}\right) & \simeq\left(\nu_{3}, \partial \nu_{3}\right)
\end{aligned}
$$

from which we have:

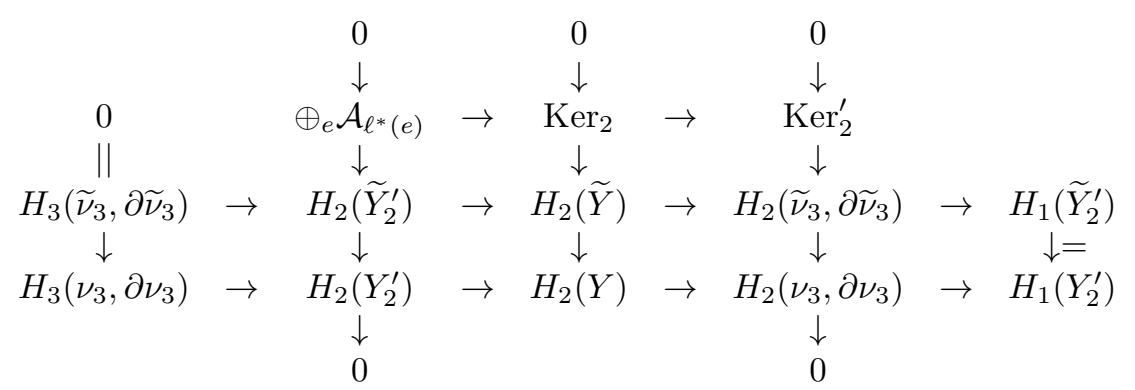

where the exactness of the second and fourth columns was established in Lemmas 3.2 and 3.3 respectively, and we take by definition $\mathrm{Ker}_{2}$ to be the kernel of $H_{2}(\widetilde{Y}) \rightarrow H_{2}(Y)$. Here Corollary 3.10 implies that $H_{3}\left(\widetilde{\nu}_{3}, \partial \widetilde{\nu}_{3}\right)=0$. By Lemma 3.2 the sequence

$$
0 \rightarrow \mathcal{A}_{\ell^{*}(e)} \rightarrow H_{2}\left(\tilde{Y}_{2}^{\prime}\right) \rightarrow H_{2}\left(Y_{2}^{\prime}\right) \rightarrow 0
$$

is exact. N.B.: We are not claiming that the top row is exact.

By Claim $3.12 H_{2}\left(\widetilde{\nu}_{3}, \partial \widetilde{\nu}_{3}\right)$ is onto. Finally, $H_{1}\left(\widetilde{Y}_{2}^{\prime}\right)=H_{1}\left(Y_{2}^{\prime}\right)$ by Lemma 3.2 A straightforward diagram chase completes the proof of Proposition 3.13 
3.7. The computation of $\rho_{*}: H_{3}(\tilde{Y}) \rightarrow H_{3}(Y)$. For each edge $e$ of $\partial \Delta$ we have the (incomplete) algebraic curve $Z(e)=Y \cap \mathcal{O}_{e}$. Its completion $\widehat{Z}(e)=Y \cap \overline{\mathcal{O}_{e}}$ is a compact complex curve. Since $\overline{\mathcal{O}_{e}}$ has singularities at most at isolated points, $\widehat{Z}(e)$ is a smooth riemann surface. Recall that for each edge $e$ of $\partial \Delta$ and each lattice point $\ell \in e^{\circ}$ we have a ruled surface $R(\ell)$ over $\widehat{Z}(e)$. The generic fiber of $R(\ell) \rightarrow \widehat{Z}(e)$ is a smooth rational curve denoted $\mathbb{P}^{1}(\ell)$. We also have $H_{3}(R(\ell))=$ $H_{1}(\widehat{Z}(e)) \otimes H_{2}\left(\mathbb{P}^{1}(\ell)\right)$. Taking the union over all $\ell \in \stackrel{\circ}{e}$ gives a surface whose $H_{3}$ is identified with $H_{1}(\widehat{Z}(e)) \otimes \mathcal{A}_{\ell^{*}(e)}$.

Here we shall prove:

Proposition 3.14. (1) The kernel of $\rho_{*}: H_{3}(\widetilde{Y}) \rightarrow H_{3}(Y)$ is identified with $\bigoplus_{e} H_{1}(\widehat{Z}(e)) \otimes \mathcal{A}_{\ell^{*}(e)}$ given by the inclusion of the ruled surfaces $\amalg_{e ; \ell \in e} R(\ell)$

$$
\bigoplus_{e ; \ell \in \stackrel{\circ}{\circ}} H_{3}(R(\ell)) \rightarrow H_{3}(\widetilde{Y}) .
$$

(2) The image of $\rho_{*}: H_{3}(\widetilde{Y}) \rightarrow H_{3}(Y)$ is Image $\left(H_{3}\left(Y_{2}\right) \rightarrow H_{3}(Y)\right)$.

Proof. First let us consider the kernel of the map $\rho_{*}: H_{3}(\widetilde{Y}) \rightarrow H_{3}(Y)$. Consider the long exact commutative diagram associated to the pairs

$$
\begin{aligned}
\widetilde{\nu}_{3} \subset \widetilde{Y} \rightarrow\left(\widetilde{Y}, \widetilde{\nu}_{3}\right) & \simeq\left(\widetilde{Y}_{2}^{\prime}, \partial \widetilde{\nu}_{3}\right) \\
\nu_{3} \subset Y \rightarrow\left(Y, \nu_{3}\right) & \simeq\left(Y_{2}^{\prime}, \partial \nu_{3}\right) .
\end{aligned}
$$

We obtain

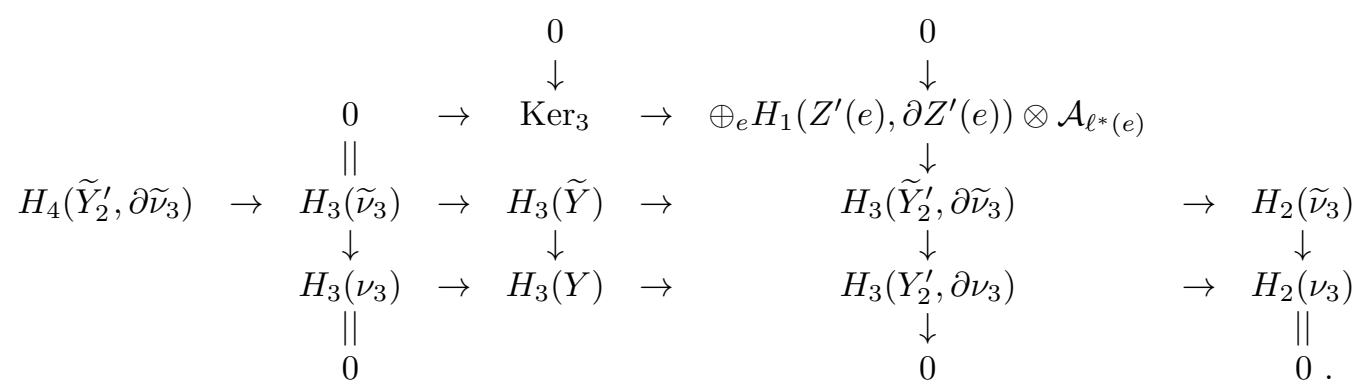

The short exact sequence in the fourth column is established in Proposition 3.4 Corollary 3.10 shows that $H_{3}\left(\widetilde{\nu}_{3}\right)=0$. Of course, since $\nu_{3}$ is a disjoint union of cones, its third homology vanishes.

Now a diagram chase shows that $\operatorname{Ker}_{3}=\operatorname{Ker}\left(\rho_{*}: H_{3}(\widetilde{Y}) \rightarrow H_{3}(Y)\right)$ sits in the sequence

$$
0 \rightarrow \mathrm{Ker}_{3} \rightarrow \oplus_{e} H_{1}\left(Z_{e}, \partial Z_{e}\right) \otimes \mathcal{A}_{\ell^{*}(e)} \stackrel{\partial}{\rightarrow} H_{2}\left(\widetilde{\nu}_{3}\right) .
$$

The following lemma completes the computation of $\mathrm{Ker}_{3}$.

Lemma 3.15. The natural map

$$
\coprod_{e ; \ell \in \stackrel{\ominus}{ }} R(\ell) \rightarrow \tilde{Y}
$$


induces an isomorphism from

$$
\bigoplus_{e ; \ell \in \stackrel{\circ}{ }} H_{3}(R(\ell)) \stackrel{\simeq}{\longrightarrow} \operatorname{Ker}_{3}
$$

PROOF. The "boundary morphism" $\partial$ in Equation 3.2 is the composition of natural homomorphisms. The first of these

$$
\bigoplus_{e} H_{1}\left(Z^{\prime}(e), \partial Z^{\prime}(e)\right) \otimes \mathcal{A}_{\ell^{*}(e)} \rightarrow \bigoplus_{e} H_{0}\left(\partial Z^{\prime}(e)\right) \otimes \mathcal{A}_{\ell^{*}(e)}
$$

is given by the direct sum of the boundary homomorphism in relative homology on the first factor tensored with the identity homomorphism on the $\mathcal{A}_{\ell^{*}(e)}$ term. The group

$$
\begin{aligned}
\bigoplus_{e} H_{0}\left(\partial Z^{\prime}(e)\right) \otimes \mathcal{A}_{\ell^{*}(e)} & =\bigoplus_{e} \bigoplus_{e \prec f} H_{0}\left(\mathcal{O}_{f} \cap Y\right) \otimes \mathcal{A}_{\ell^{*}(e)} \\
& =\bigoplus_{f} \bigoplus_{e \prec f} H_{0}\left(\mathcal{O}_{f} \cap Y\right) \otimes \mathcal{A}_{\ell^{*}(e)} \\
& =\bigoplus_{f} H_{0}\left(\mathcal{O}_{f} \cap Y\right) \otimes \bigoplus_{e \prec f} \mathcal{A}_{\ell^{*}(e)}
\end{aligned}
$$

where the first isomorphism follows from the fact that $\partial Z^{\prime}(e)=\coprod_{e \prec f}\left(\mathcal{O}_{f} \cap Y\right) \times S^{1}$. The second homomorphism

$$
\bigoplus_{f}\left[H_{0}\left(\mathcal{O}_{f} \cap Y\right) \otimes \bigoplus_{e \prec f} \mathcal{A}_{\ell^{*}(e)}\right] \rightarrow H_{2}\left(\widetilde{\nu}_{3}\right)=\bigoplus_{f}\left[H_{0}\left(\mathcal{O}_{f} \cap Y\right) \otimes H_{2}(\mathcal{S}(f))\right]
$$

is given by $\bigoplus_{f} \operatorname{Id}_{H_{0}\left(\mathcal{O}_{f} \cap Y\right)} \otimes\left[\bigoplus_{e \prec f} \mathcal{A}_{\ell^{*}(e)} \rightarrow H_{2}(\mathcal{S}(f))\right]$. The main point is to show the injectivity of this last morphism. That is to say, for all $f$, the map $\bigoplus_{e \prec f} \mathcal{A}_{\ell^{*}(e)} \rightarrow H_{2}(\mathcal{S}(f))$ is injective. In light of the isomorphism $H_{2}(\mathcal{S}(f)) \simeq$ $H_{2}\left(\widetilde{\nu}_{3}(f)\right)$, and the fact that the morphism $\bigoplus_{e \prec f} \mathcal{A}_{\ell^{*}(e)} \rightarrow H_{2}(\mathcal{S}(f))$ factors through the morphism $H_{2}\left(\partial \widetilde{\nu}_{3}(f)\right) \rightarrow H_{2}(\mathcal{S}(f))$, which is itself injective by the long exact sequence of the pair (the previous term being $H_{3}\left(\widetilde{\nu}_{3}, \partial \widetilde{\nu}_{3}\right)=0$ ), it suffices to show that $\bigoplus_{e \prec f} \mathcal{A}_{\ell^{*}(e)} \rightarrow H_{2}\left(\partial \widetilde{\nu}_{3}(f)\right)$ is injective. This last follows by Lemma 3.3. Thus $K$ is the kernel of the homomorphism in Equation 3.3 This latter kernel is easily identified with $\oplus_{e} H_{1}(\widehat{Z}(e)) \otimes \mathcal{A}_{\ell^{*}(e)}$ which is naturally identified with

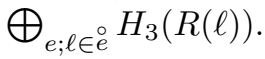

Now, let's compute the image of $\rho_{*}: H_{3}(\widetilde{Y}) \rightarrow H_{3}(Y)$. We have the long exact commutative diagram associated to the sequences

$$
\begin{aligned}
& \widetilde{Y}_{2} \subset \widetilde{Y}_{3} \rightarrow\left(\widetilde{Y}_{3}, \widetilde{Y}_{2}\right) \simeq\left(\widetilde{\nu}_{3}, \partial \widetilde{\nu}_{3}\right) \\
& Y_{2} \subset Y_{3} \rightarrow\left(Y_{3}, Y_{2}\right) \simeq\left(\nu_{3}, \partial \nu_{3}\right)
\end{aligned}
$$


We obtain

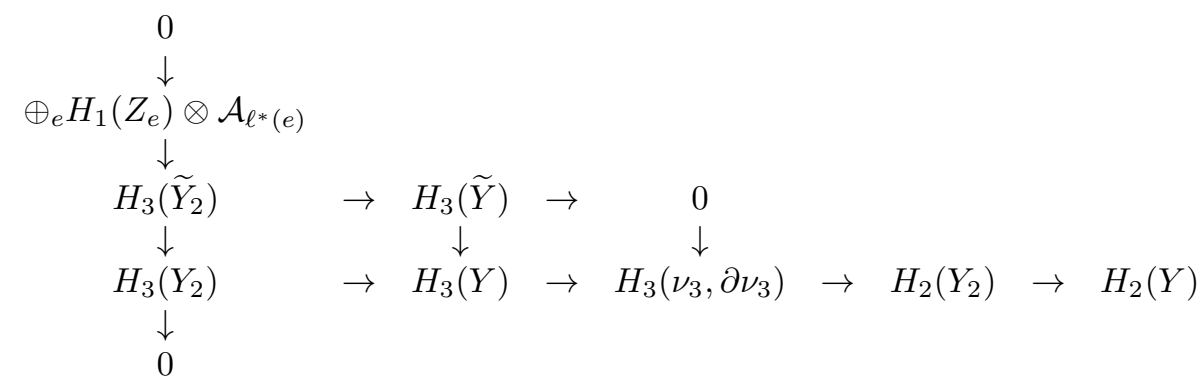

yielding

$$
\operatorname{Image}\left(H_{3}(\widetilde{Y}) \rightarrow H_{3}(Y)\right)=\operatorname{Image}\left(H_{3}\left(Y_{2}\right) \rightarrow H_{3}(Y)\right)
$$

It is not true in general that $\rho_{*}: H_{3}(\widetilde{Y}) \rightarrow H_{3}(Y)$ is onto, or equivalently that $H_{3}\left(Y_{2}\right) \rightarrow H_{3}(Y)$ is onto. From the exact sequence of the pair $\left(Y, Y_{2}\right)$ and the fact that $\left(Y, Y_{2}\right)$ is excisively equivalent to $\left(\nu_{3}, \partial \nu_{3}\right)$ we see that the cokernel of $H_{3}\left(Y_{2}\right) \rightarrow H_{3}(Y)$ is identified with the kernel of $H_{3}\left(\nu_{3}, \partial \nu_{3}\right) \rightarrow H_{2}\left(Y_{2}\right)$. Of course, since each component of $\nu_{3}$ is a cone, this kernel is identified with the kernel of the map induced by the inclusion $H_{2}\left(\partial \nu_{3}\right) \rightarrow H_{2}\left(Y_{2}\right)$. Let's compute rationally. Arguing as in the proof of Lemma 2.21 using $Y_{0} \subset Y_{1} \subset Y_{2}$ we see that $H_{1}\left(Y_{2} ; \mathbb{Q}\right)=0$ and

$$
H_{2}\left(Y_{2} ; \mathbb{Q}\right)=\operatorname{Ker}\left(\oplus_{v \in V}(\Delta) \mathbb{Z}\langle v\rangle \rightarrow N \otimes \mathbb{Q}\right) .
$$

Clearly, this map is onto rationally and hence the rank of $H_{2}\left(Y_{2}\right)$ is $\# V(\Delta)-n$. For each two-face $f$ of $\partial \Delta$, the boundary of the regular neighborhood $\partial \nu(f)$ is homotopy equivalent to $V(f)^{*}$, the complement of the fixed point $0_{f}$ in $V(f)$. Similar arguments to the ones for $Y_{2}$ show that the rank of $H_{2}\left(V(f)^{*}, \mathbb{Q}\right)$ is given by the number of vertices of $f$ minus 3 . (Indeed the map between these groups is easily determined from the combinatorial configuration of vertices and two-faces.) In any event if the sum over the two-faces of the number of vertices of the face minus three is greater than the number of vertices of $\Delta$ minus $n$, then the kernel of this map is non-trivial and hence $H_{3}(\widetilde{Y}) \rightarrow H_{3}(Y)$ will not be onto. As an explicit example where this map is not onto, we have the 4-cube which has 24 twofaces, each with four vertices. For each two-face $f$ the affine three-fold $V(f)$ is a (complex) cone on the quotient of $\mathbb{P}^{1} \times \mathbb{P}^{1}$ by an involution preserving the factors. Thus, $V(f)^{*}$ has second homology of rank 1 . The cube itself has 16 vertices so that $H_{2}\left(Y_{2}\right)$ has rank 12. Hence, in this case the kernel of the map has rank at least 12, and in fact has rank 13.

COROLlary 3.16. In the hypersurface case the rank of the kernel of $\rho_{*}: H_{3}(\widetilde{Y}) \rightarrow$ $H_{3}(Y)$ is

$$
2 \sum_{e \in E} \ell^{*}(e) \ell^{*}\left(e^{\vee}\right)
$$


For complete intersections of ample divisors the rank of the kernel of the map $\rho_{*}: H_{3}(\widetilde{Y}) \rightarrow H_{3}(Y)$ is given by

$$
2 \sum_{e \in E} \ell^{*}(e)\left(\sum_{J \subset I ; J \neq \emptyset}(-1)^{3-|J|} \ell^{*}\left(\sum_{j \in J} e_{j}^{\vee}\right)\right) .
$$

In both cases the Hodge structure on this kernel is of type $(2,1)$ and $(1,2)$ each piece being of half the rank.

Proof. According to Corollary 2.18 in the hypersurface case the rank of $H_{1}(\widehat{Z}(e))$ is $2 \ell^{*}\left(e^{\vee}\right)$. According to Corollary [2.20 the formula for the rank of $H_{1}(\widehat{Z}(e))$ in the general case is

$$
2 \sum_{J \subset I ; J \neq \emptyset}(-1)^{3-|J|} \ell^{*}\left(\sum_{j \in J} e_{j}^{\vee}\right) .
$$

The result is immediate from this.

\subsection{The tangent space to the space of polynomial deformations.}

Lemma 3.17. Suppose $M$ is a smooth n-dimensional Calabi-Yau manifold and let $D \subset M$ be a smooth divisor. Suppose the pair $(M, D)$ deforms. Let

$$
\alpha_{M} \in H^{1}\left(M, T_{M}\right)=H^{n-1,1}(M)
$$

be the Kodaira-Spencer class of the deformation of $M$. Then the restriction of $\alpha_{M}$ to a class in $H^{n-1,1}(D)$ vanishes.

Proof. Suppose we have a deformation of the pair $(M, D)$. We have the following sequence in cohomology

$$
\begin{gathered}
\left.H^{0}\left(D, \nu_{D \subset M}\right) \rightarrow H^{1}\left(D, T_{D}\right) \rightarrow \quad \begin{array}{c}
H^{1}\left(D,\left.T_{M}\right|_{D}\right) \\
\uparrow \\
H^{1}\left(M, T_{M}\right)
\end{array}\right) H^{1}\left(D, \nu_{D \subset M}\right)
\end{gathered}
$$

where, via the Kodaira-Spencer mapping, $H^{1}\left(M, T_{M}\right)$ corresponds to the tangent space to deformations of $M$ and $H^{1}\left(D, T_{D}\right)$ to those of $D$. Deformations of the pair $(M, D)$ are given by a pair of classes $\alpha_{M} \in H^{1}\left(M, T_{M}\right)$ and $\alpha_{D} \in H^{1}\left(D, T_{D}\right)$ with the same image $\alpha^{\prime} \in H^{1}\left(D,\left.T_{M}\right|_{D}\right)$. Thus, the composite mapping

$$
H^{1}\left(M, T_{M}\right) \rightarrow H^{1}\left(D,\left.T_{M}\right|_{D}\right)
$$

sends $\alpha_{M}$ to zero. Moreover, we have the sequence

$$
\begin{aligned}
& H^{1}\left(M, \Omega_{M}^{n-1}\right) \rightarrow H^{1}\left(D,\left.\Omega_{M}^{n-1}\right|_{D}\right) \rightarrow H^{1}\left(D, \Omega_{D}^{n-1}\right) \\
& H^{1}\left(M, T_{M}\right) \quad H^{1}\left(D, K_{D}\right)
\end{aligned}
$$

so that the composition map

$$
H^{1}\left(M, T_{M}\right) \rightarrow H^{1}\left(D,\left.T_{M}\right|_{D}\right) \rightarrow H^{1}\left(D, T_{D}\right)
$$

is identified with the natural restriction map

$$
H^{n-1,1}(M) \rightarrow H^{n-1,1}(D)
$$

This shows that if the pair $(M, D)$ deforms, then the restriction map on $H^{n-1,1}$ is trivial. Conversely, if the image of $\alpha_{M}$ in $H^{1}\left(D,\left.T_{M}\right|_{D}\right)$ goes to zero in $H^{1}\left(D, \nu_{D \subset M}\right)$ then there is a class $\alpha_{D} \in H^{1}\left(D, T_{D}\right)$ with the same image as $\alpha_{M}$ in $H^{1}\left(D,\left.T_{M}\right|_{D}\right)$. The pair $\left(\alpha_{M}, \alpha_{D}\right)$ then gives an infinitesimal deformation of $(M, D)$. 
Now let us apply this to the situation of Calabi-Yau threefolds in toric varieties.

Corollary 3.18. Let $\alpha \in H^{1}(\widetilde{Y}, T \widetilde{Y})=H^{2,1}(\widetilde{Y})$ be the Kodaira-Spencer class of a deformation of $\widetilde{Y}$ induced by taking the preimage under the map $\rho: \mathbb{P}_{\mathcal{T}} \rightarrow \mathbb{P}_{\Delta}$ of a polynomial deformation of $Y \subset X$ (i.e., a deformation obtained by varying the coefficients of the polynomials cutting out $Y$ as a complete intersection). Then for each edge $e$ of $\partial \Delta$ and any lattice point $\ell \in \stackrel{\circ}{e}$, the element $\alpha \in H^{2,1}(\widetilde{Y})$ restricts to zero in $H^{2,1}(R(\ell))$.

Proof. Since the deformation $\left\{Y_{t}\right\}$ of $Y$ takes place in $\mathbb{P}_{\Delta}$ we have the family of curves $\widehat{Z}_{t}(e)=Y_{t} \cap \overline{\mathcal{O}}_{e}$ and their preimages which are surfaces $R_{t}(\ell) \subset \widetilde{Y}_{t}$ deforming $R(\ell)$. Thus, the given deformation of $\widetilde{Y}$ lifts to a deformation of the pair $(\widetilde{Y}, R(\ell))$. Applying the previous lemma gives the result.

COROLlary 3.19. Let $H_{\text {poly }}^{2,1}(\widetilde{Y})$ be the tangent space of the polynomial deformations of $\widetilde{Y}$ inside $H^{2,1}(\widetilde{Y})$. This subspace is identified with the kernel of the map

$$
H^{2,1}(\tilde{Y}) \rightarrow \bigoplus_{\ell \in N \cap \partial \Delta^{(1)} \backslash V(\Delta)} H^{2,1}(R(\ell))
$$

Proof. The previous result shows that $H_{\text {poly }}^{2,1}(\widetilde{Y})$ is contained in the kernel of the restriction mapping. In Corollary 3.16 we showed that the rank of the kernel of the restriction mapping is equal to the rank of the space of non-polynomial deformations (i.e., the correction term) as computed by $\mathbf{B B}$.

\section{4. $K^{0}$ and $K^{1}$ of Calabi-Yau threefolds in terms of homology}

The 7-dimensional stage of the Postnikov tower for $B S U$, denoted $B S U^{(7)}$, contains the homotopy groups $\pi_{i}(B S U)$ for $i \leq 7$ and has trivial groups in degrees $\geq 8$. In particular,

$$
\pi_{i}\left(B S U^{(7)}\right) \simeq \begin{cases}\mathbb{Z} & i=4,6 \\ 0 & \text { otherwise }\end{cases}
$$

If $X$ is a $\mathrm{CW}$ complex of dimension $\leq 7$, then the natural map from

$$
[X, B S U] \rightarrow\left[X, B S U^{(7)}\right]
$$

is a bijection.

$B S U^{(7)}$ is given by a fibration

$$
K(\mathbb{Z}, 6) \rightarrow B S U^{(7)} \rightarrow K(\mathbb{Z}, 4)
$$

with $k$-invariant $\delta S q^{2} \iota_{4}$ the unique nontrivial element (of order two) in $H^{7}(K(\mathbb{Z}, 4) ; \mathbb{Z}$ ). Thus we also have a fibration

$$
B S U^{(7)} \rightarrow K(\mathbb{Z}, 4) \times K(\mathbb{Z}, 6) \rightarrow K(\mathbb{Z} / 2,6)
$$

where the first map is given by the second and third Chern classes $\left(c_{2}, c_{3}\right)$ and the second map is given by $S q^{2} \iota_{4}+\left[\iota_{6}\right]_{2}$, with $\iota_{4}$ and $\iota_{6}$ being the fundamental classes of the factors and $[\cdot]_{2}$ denoting reduction $\bmod 2$.

This means that for any CW-complex $X$ of dimension $\leq 7$ we have that

$$
[X, B S U]=\left[X, B S U^{(7)}\right]
$$


sits in an exact sequence

$H^{3}(X ; \mathbb{Z}) \oplus H^{5}(X ; \mathbb{Z}) \stackrel{\alpha}{\rightarrow} H^{5}(X ; \mathbb{Z} / 2) \rightarrow[X, B S U] \rightarrow H^{4}(X ; \mathbb{Z}) \oplus H^{6}(X ; \mathbb{Z}) \stackrel{\beta}{\rightarrow} H^{6}(X ; \mathbb{Z} / 2)$

where $\alpha(a, b)=S q^{2} a+[b]_{2}$ and $\beta(c, d)=S q^{2} c+[d]_{2}$. In the special case that $M$ is a closed, oriented 6 -manifold, $H^{6}(M ; \mathbb{Z})$ is torsion-free, and hence the mod 2 reduction $H^{5}(M ; \mathbb{Z}) \rightarrow H^{5}(M ; \mathbb{Z} / 2)$ is onto. Thus, we have

$$
0 \rightarrow[M, B S U] \stackrel{\left(c_{2}, c_{3}\right)}{\rightarrow} H^{4}(M ; \mathbb{Z}) \oplus H^{6}(M ; \mathbb{Z}) \rightarrow H^{6}(M ; \mathbb{Z} / 2) \rightarrow 0 .
$$

This proves

Lemma 4.1. Let $M$ be a closed oriented 6-manifold. Then $[M, B S U]$ is isomorphic to

$$
\left\{\left(c_{2}, c_{3}\right) \in H^{4}(M ; \mathbb{Z}) \oplus H^{6}(M ; \mathbb{Z}) \mid S q^{2} c_{2}=\left[c_{3}\right]_{2}\right\}
$$

where the isomorphism is given by taking the 2nd and 3rd Chern class.

An examination of low dimensional examples allows one to extend this result to $\widetilde{K^{0}}(M)=[M, B U]$ :

LEMmA 4.2. Let $M$ be a closed oriented 6-manifold. Then $\widetilde{K^{0}}(M)$ is isomorphic to

$\left\{\left(c_{1}, c_{2}, c_{3}\right) \in H^{2}(M ; \mathbb{Z}) \oplus H^{4}(M ; \mathbb{Z}) \oplus H^{6}(M ; \mathbb{Z}) \mid S q^{2} c_{2}=\left[c_{3}\right]_{2}+c_{1} c_{2}+c_{1}^{3}\right\}$

where the isomorphism is given by taking the 1st, 2nd, and 3rd Chern class.

Corollary 4.3. If $M$ is a Calabi-Yau threefold, then

$$
\text { Tor } K^{0}(M)=\text { Tor } H^{2}(M ; \mathbb{Z}) \oplus \text { Tor } H^{4}(M ; \mathbb{Z})
$$

and

$$
\operatorname{rank} K^{0}(M)=\sum_{* \leq 3} \operatorname{rank}\left(H^{2 *}(M ; \mathbb{Z}) / \text { Torsion }\right) .
$$

PROOF. The rank statement is well-known. The torsion statement follows from the above since

$$
S q^{2}: H^{4}(M ; \mathbb{Z}) \rightarrow H^{6}(M ; \mathbb{Z})
$$

is zero. This follows from the fact that this map is the cup product with $w_{2}(M)$ which, for any almost complex manifold, is the reduction $\bmod 2$ of $c_{1}(M)$. The Calabi-Yau condition means that $c_{1}(M)=0$. is

In other words, we have that, for a Calabi-Yau threefold $M$, the even K-group

$$
K^{0}(M) \simeq \mathbb{Z} \oplus H^{2}(M ; \mathbb{Z}) \oplus H^{4}(M ; \mathbb{Z}) \oplus 2 \cdot H^{6}(M ; \mathbb{Z}) .
$$

Similar arguments show that

Lemma 4.4. Let $M$ be a closed oriented 6 -manifold. Then $K^{1}(M)=\widetilde{K^{0}}(\Sigma M)$ is

$$
H^{1}(M ; \mathbb{Z}) \times\left[\Sigma M, B S U^{(7)}\right]
$$

and we have an exact sequence

$$
0 \rightarrow H^{5}(M ; \mathbb{Z}) \rightarrow\left[\Sigma M, B S U^{(7)}\right] \rightarrow H^{3}(M ; \mathbb{Z}) \rightarrow 0
$$

coming from the fibration 4.1).

Corollary 4.5. When $M$ is a Calabi-Yau threefold the extension class is trivial. 
Proof. Once again

$$
S q^{2}: H^{3}(M ; \mathbb{Z}) \rightarrow H^{5}(M ; \mathbb{Z} / 2)
$$

is the reduction mod 2 of cupping with $c_{1}$, which is consequently trivial. Thus, by Lemma 4.2 for any class $a \in H^{3}(M ; \mathbb{Z}$ ) there is a unique (up to isomorphism) bundle over $\Sigma M$ with $c_{2}=a$ and $c_{1}=c_{3}=0$. The Whitney sum formula shows that these bundles form a subgroup in $\widetilde{K^{0}}(\Sigma M)$, and hence this construction gives a splitting of the sequence.

In other words, we have that, for a Calabi-Yau threefold $M$, the odd K-group is

$$
K^{1}(M) \simeq H^{1}(M ; \mathbb{Z}) \oplus H^{3}(M ; \mathbb{Z}) \oplus H^{5}(M ; \mathbb{Z}) .
$$

\section{References}

[Bat] Batyrev, V.V. "Dual Polyhedra and Mirror Symmetry for Calabi-Yau Hypersurfaces in Toric Varieties." J. Algebraic Geom. 3, no. 3, 493-535 (1994).

[BB] Batyrev, V.V. and L. Borisov. "On Calabi-Yau Complete Intersections in Toric Varieties." Higher-dimensional Complex Varieties (Trento, 1994), 39-65 (1996).

[BK] Batyrev, V.V. and M. Kreuzer. "Integral Cohomology and Mirror Symmetry for CalabiYau 3-Folds." To appear in Mirror Symmetry V, Proceedings of the BIRS workshop on Calabi-Yau Varieties and Mirror Symmetry, December 6-11, 2003.

[CK] Cox, D. and S. Katz. Mirror Symmetry and Algebraic Geometry. Mathematical Surveys and Monographs, 68 (1999).

[DK] Danilov, V.I. and A.G. Khovanskii. "Newton Polyhedra and an Algorithm for Computing Hodge-Deligne Numbers," Math. USSR Izvestiya, Vol. 29 (1987), No. 2, 279-298.

[Del] Deligne, P. "Théorie de Hodge. III." Inst. Hautes Études Sci. Publ. Math. No. 44, (1974), $5-77$.

[DM] Doran, C. and J. Morgan. "Mirror Symmetry and Integral Variations of Hodge Structure Underlying One Parameter Families of Calabi-Yau Threefolds." To appear in Mirror Symmetry V, Proceedings of the BIRS workshop on Calabi-Yau Varieties and Mirror Symmetry, December 6-11, 2003.

[Ful] Fulton, W. "Introduction to Toric Varieties," Annals of Mathematics Studies, Study 131 (1993).

[Kho] Khovanskii, A.G. "Newton Polyhedra and the Genus of Complete Intersections," Functional Anal. Appl., Volume 12, Number 1, pages 38-46 (1978).

[KS] Kreuzer, M. and H. Skarke. "Complete classification of Reflexive Polyhedra in Four Dimensions." Adv. Theor. Math. Phys. 4, no. 6, 1209-1230 (2000).

Department of Mathematics, University of Washington, Seattle, Washington 98195

E-mail address: doran@math.washington.edu

Department of Mathematics, Columbia University, New York, New York 10027

E-mail address: jm@math.columbia.edu 